\title{
A topologically minimal, weakly reducible, unstabilized Heegaard splitting of genus three is critical
}

\author{
JUNGSOO KIM
}

Let $(\mathcal{V}, \mathcal{W} ; F)$ be a weakly reducible unstabilized genus three Heegaard splitting in an orientable, irreducible 3-manifold $M$. In this article, we prove that either the disk complex $\mathcal{D}(F)$ is contractible or $F$ is critical. Hence, the topological index of $F$ is 2 if $F$ is topologically minimal.

$57 \mathrm{M} 50$

\section{Introduction and result}

Throughout this paper, all surfaces and 3-manifolds will be taken to be compact and orientable. Bachman introduced the concept of a critical surface [1] and proved that a critical surface intersects an incompressible surface such that the intersection is essential on both surfaces up to isotopy in an irreducible manifold. A strongly irreducible Heegaard surface also has this property, as seen in Schultens [19]. In a subsequent paper [2], he generalized the definition of critical surface for the proof of Gordon's conjecture using the notation from the standard disk complex. Moreover, he defined the concept of a topologically minimal surface, which includes incompressible surfaces, strongly irreducible surfaces, critical surfaces, and so on; the topologically minimal surfaces are distinguished by the topological index [3]. Indeed, he proved that a topologically minimal surface also intersects an incompressible surface such that the intersection is essential on both surfaces up to isotopy in an irreducible manifold. He also found counterexamples to the stabilization conjecture using this concept [7]. In a series of articles $[4 ; 5 ; 6]$, he proved that there is a resemblance between a topologically minimal surface and a geometrically minimal surface.

In recent results, including the author's works, several examples of critical Heegaard surfaces were found and most of them are easily constructible; see Bachman and Johnson [8], Lee [14], Lei and Qiang [9], and Kim [12; 13]. Hence, it is now conjectured that it is more common for a weakly reducible surface to be topologically minimal than not. Indeed, the condition that the disk complex is noncontractible for a topologically minimal surface seems to be more common than the contrary.

In this article, we will prove the following theorem, giving evidence for an affirmative answer to this question. 
Theorem 1.1 Let $(\mathcal{V}, \mathcal{W} ; F)$ be a weakly reducible unstabilized genus three Heegaard splitting in an orientable, irreducible 3-manifold $M$. If every weak reducing pair of $F$ gives the same generalized Heegaard splitting after weak reduction, and the embedding of each thick level in the relevant compression body is unique up to isotopy, then the disk complex $\mathcal{D}(F)$ is contractible. Otherwise, $F$ is critical.

Note that the author proved that if a weakly reducible unstabilized genus-three Heegaard splitting in an orientable, irreducible 3-manifold is topologically minimal, then the topological index is at most 4 [12], and Theorem 1.1 improves the upper bound of the topological index, that is, the topological index is 2 if $F$ is topologically minimal. Since there exist many unstabilized critical Heegaard surfaces of genus three, this upper bound is sharp.

Acknowledgments The author gives thanks to the referee for a number of helpful suggestions for improvement in the article. The author is grateful to Professor Jesse Johnson for his interest in my result and handling the article, and to Professor Jongil Park and the other professors of the BK21 PLUS SNU Mathematical Sciences Division for giving me the opportunity to be a postdoctoral researcher. This research was supported by BK21 PLUS SNU Mathematical Sciences Division.

\section{Preliminaries}

Definition 2.1 A compression body is a 3-manifold which can be obtained by starting with a closed orientable connected surface $F$, forming the product $F \times I$, attaching some number of 2-handles to $F \times\{1\}$, and capping off all resulting 2 -sphere boundary components that are not contained in $F \times\{0\}$ with 3 -balls. The boundary component $F \times\{0\}$ is referred to as $\partial_{+}$. The rest of the boundary is referred to as $\partial_{-}$.

Definition 2.2 A Heegaard splitting of a 3-manifold $M$ is an expression of $M$ as a union $\mathcal{V} \cup_{F} \mathcal{W}$, denoted as $(\mathcal{V}, \mathcal{W} ; F)$ (or $(\mathcal{V}, \mathcal{W})$ if necessary), where $\mathcal{V}$ and $\mathcal{W}$ are compression bodies that intersect in a transversally oriented surface $F=\partial_{+} \mathcal{V}=\partial_{+} \mathcal{W}$. We say $F$ is the Heegaard surface of this splitting. If $\mathcal{V}$ or $\mathcal{W}$ is homeomorphic to a product, then we say the splitting is trivial. If there are compressing disks $V \subset \mathcal{V}$ and $W \subset \mathcal{W}$ such that $V \cap W=\varnothing$, then we say the splitting is weakly reducible and call the pair $(V, W)$ a weak reducing pair. If $(V, W)$ is a weak reducing pair and $\partial V$ is isotopic to $\partial W$ in $F$, then we call $(V, W)$ a reducing pair. If the splitting is not trivial and we cannot take a weak reducing pair, then we call the splitting strongly irreducible. If there is a pair of compressing disks $(\bar{V}, \bar{W})$ such that $\bar{V}$ intersects $\bar{W}$ transversely in a point in $F$, then we call this pair a canceling pair and say the splitting is stabilized. Otherwise, we say the splitting is unstabilized. 
Definition 2.3 Let $F$ be a surface of genus at least 2 in a compact, orientable 3manifold $M$. Then the disk complex $\mathcal{D}(F)$ is defined as follows:

(1) Vertices of $\mathcal{D}(F)$ are isotopy classes of compressing disks for $F$.

(2) A set of $m+1$ vertices forms an $m$-simplex if there are representatives for each that are pairwise disjoint.

Definition 2.4 (Bachman [3]) The homotopy index of a complex $\Gamma$ is defined to be 0 if $\Gamma=\varnothing$, and defined to be $n$ for the smallest $n$ such that $\pi_{n-1}(\Gamma)$ is nontrivial, otherwise. We say a separating surface $F$ with no torus components is topologically minimal if its disk complex $\mathcal{D}(F)$ is either empty or noncontractible. When $F$ is topologically minimal, we say its topological index is the homotopy index of $\mathcal{D}(F)$. If $F$ is topologically minimal and its topological index is 2, then we call $F$ a critical surface.

Note that Bachman originally defined a critical surface in a different way in [2] and proved its equivalence to being index-two in [3].

Definition 2.5 Consider a Heegaard splitting $(\mathcal{V}, \mathcal{W} ; F)$ of an orientable, irreducible 3-manifold $M$. Let $\mathcal{D}_{\mathcal{V}}(F)$ and $\mathcal{D}_{\mathcal{W}}(F)$ be the subcomplexes of $\mathcal{D}(F)$ spanned by compressing disks in $\mathcal{V}$ and $\mathcal{W}$, respectively; see [16, Section 5]. We call these subcomplexes the disk complexes of $\mathcal{V}$ and $\mathcal{W}$. Let $\mathcal{D}_{\mathcal{V W}}(F)$ be the subset of $\mathcal{D}(F)$ consisting of the simplices with at least one vertex from $\mathcal{D}_{\mathcal{V}}(F)$ and at least one vertex from $\mathcal{D}_{\mathcal{W}}(F)$.

Theorem 2.6 (McCullough [16, Theorem 5.3]) $\mathcal{D}_{\mathcal{V}}(F)$ and $\mathcal{D}_{\mathcal{W}}(F)$ are contractible.

Note that $\mathcal{D}(F)=\mathcal{D}_{\mathcal{V}}(F) \cup \mathcal{D}_{\mathcal{V W}}(F) \cup \mathcal{D}_{\mathcal{W}}(F)$.

From now on, we will consider only unstabilized Heegaard splittings of an irreducible 3-manifold. If a Heegaard splitting of a compact 3-manifold has a reducing pair, then the manifold is reducible or the splitting is stabilized; see Saito, Scharlemann and Schultens [17]. Hence, we can exclude the possibilities of reducing pairs among weak reducing pairs.

Definition 2.7 Suppose $W$ is a compressing disk for $F \subset M$. Then there is a subset of $M$ that can be identified with $W \times I$ so that $W=W \times\left\{\frac{1}{2}\right\}$ and $F \cap(W \times I)=$ $(\partial W) \times I$. We form the surface $F_{W}$, obtained by compressing $F$ along $W$, by removing $(\partial W) \times I$ from $F$ and replacing it with $W \times(\partial I)$. We say the two disks $W \times(\partial I)$ in $F_{W}$ are the scars of $W$. 
Lemma 2.8 (Lustig and Moriah [15, Lemma 1.1]) Suppose that $M$ is an irreducible 3 -manifold and $(\mathcal{V}, \mathcal{W} ; F)$ is an unstabilized Heegaard splitting of $M$. If $F^{\prime}$ is obtained by compressing $F$ along a collection of pairwise disjoint disks, then no $S^{2}$ component of $F^{\prime}$ can have scars from disks in both $\mathcal{V}$ and $\mathcal{W}$.

Lemma 2.9 (Kim [12, Lemma 2.9]) Suppose that $M$ is an irreducible 3-manifold and $(\mathcal{V}, \mathcal{W} ; F)$ is an unstabilized genus-three Heegaard splitting of $M$. If there exist three mutually disjoint compressing disks $V, V^{\prime} \subset \mathcal{V}$ and $W \subset \mathcal{W}$, then either $V$ is isotopic to $V^{\prime}$ in $\mathcal{V}$, or one of $\partial V$ and $\partial V^{\prime}$ bounds a punctured torus $T$ in $F$ and the other is a nonseparating loop in $T$. Moreover, we cannot choose three weak reducing pairs $\left(V_{0}, W\right),\left(V_{1}, W\right)$, and $\left(V_{2}, W\right)$ such that $V_{i}$ and $V_{j}$ are mutually disjoint and nonisotopic in $\mathcal{V}$ for $i \neq j$.

The next is the definition of generalized Heegaard splitting originated from Scharlemann and Thompson [18].

Definition 2.10 (Bachman [2, Definition 4.1]) A generalized Heegaard splitting $(G H S) \boldsymbol{H}$ of a 3-manifold $M$ is a pair of sets of pairwise disjoint, transversally oriented, connected surfaces, Thick $(\boldsymbol{H})$ and Thin $(\boldsymbol{H})$ (called the thick levels and thin levels, respectively), which satisfies the following conditions:

(1) Each component $M^{\prime}$ of $M$-Thin $(\boldsymbol{H})$ meets a unique element $H_{+}$of Thick $(\boldsymbol{H})$ and $H_{+}$is a Heegaard surface in $M^{\prime}$. Henceforth we will denote the closure of the component of $M-\operatorname{Thin}(\boldsymbol{H})$ that contains an element $H_{+} \in \operatorname{Thick}(\boldsymbol{H})$ as $M\left(H_{+}\right)$.

(2) As each Heegaard surface $H_{+} \subset M\left(H_{+}\right)$is transversally oriented, we can consistently talk about the points of $M\left(H_{+}\right)$that are "above" or "below" $H_{+}$. Suppose $H_{-} \in \operatorname{Thin}(\boldsymbol{H})$. Let $M\left(H_{+}\right)$and $M\left(H_{+}^{\prime}\right)$ be the submanifolds on each side of $H_{-}$. Then $H_{-}$is below $H_{+}$if and only if it is above $H_{+}^{\prime}$.

(3) There is a partial ordering on the elements of $\operatorname{Thin}(\boldsymbol{H})$ which satisfies the following: suppose $H_{+}$is an element of $\operatorname{Thick}(\boldsymbol{H}), H_{-}$is a component of $\partial M\left(H_{+}\right)$above $H_{+}$and $H_{-}^{\prime}$ is a component of $\partial M\left(H_{+}\right)$below $H_{+}$; then $H_{-}>H_{-}^{\prime}$.

We denote the maximal subset of Thin $(\boldsymbol{H})$ consisting of surfaces only in the interior of $M$ as $\overline{\operatorname{Thin}}(\boldsymbol{H})$ and call it the inner thin level. A GHS in this article is the same as a pseudo-GHS in [2] since we allow a GHS to have product compression bodies and we do not encounter thin 2-spheres. 


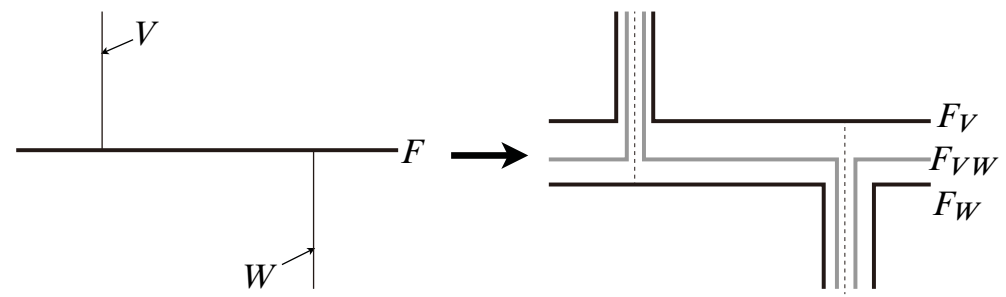

Figure 1: Preweak reduction.

Definition 2.11 (Bachman [2, Definitions 5.2, 5.3 and 5.6]) Let $M$ be a compact, orientable 3-manifold. Let $\boldsymbol{H}$ be a Heegaard splitting of $M$, that is, Thick $(\boldsymbol{H})=\{F\}$ and Thin $(\boldsymbol{H})$ consists of $\partial M$. Let $V$ and $W$ be disjoint compressing disks of $F$ from the opposite sides of $F$ such that $F_{V W}$ has no 2-sphere component. (Lemma 2.8 guarantees that $F_{V W}$ will not have a 2 -sphere component in the proof of Theorem 1.1.) Define

$$
\begin{aligned}
\operatorname{Thick}\left(\boldsymbol{G}^{\prime}\right) & =(\operatorname{Thick}(\boldsymbol{H})-\{F\}) \cup\left\{F_{V}, F_{W}\right\}, \\
\operatorname{Thin}\left(\boldsymbol{G}^{\prime}\right) & =\operatorname{Thin}(\boldsymbol{H}) \cup\left\{F_{V W}\right\},
\end{aligned}
$$

where we assume that each element of $\operatorname{Thick}\left(\boldsymbol{G}^{\prime}\right)$ belongs to the interior of $\mathcal{V}$ or $\mathcal{W}$ by slightly pushing off $F_{V}$ or $F_{W}$ into the interior of $\mathcal{V}$ or $\mathcal{W}$, respectively, then also assume that they miss $F_{V W}$. We say the GHS $\boldsymbol{G}^{\prime}=\left\{\operatorname{Thick}\left(\boldsymbol{G}^{\prime}\right)\right.$, $\left.\operatorname{Thin}\left(\boldsymbol{G}^{\prime}\right)\right\}$ is obtained from $\boldsymbol{H}$ by preweak reduction along $(V, W)$. The relative position of the elements of Thick $\left(\boldsymbol{G}^{\prime}\right)$ and $\operatorname{Thin}\left(\boldsymbol{G}^{\prime}\right)$ follows the order described in Figure 1. If there are elements $S \in \operatorname{Thick}\left(\boldsymbol{G}^{\prime}\right)$ and $s \in \overline{\operatorname{Thin}}\left(\boldsymbol{G}^{\prime}\right)$ that cobound a product region $P$ of $M$ such that $P \cap \operatorname{Thick}\left(\boldsymbol{G}^{\prime}\right)=S$ and $P \cap \operatorname{Thin}\left(\boldsymbol{G}^{\prime}\right)=s$ then remove $S$ from $\operatorname{Thick}\left(\boldsymbol{G}^{\prime}\right)$ and $s$ from $\operatorname{Thin}\left(\boldsymbol{G}^{\prime}\right)$. If we repeat this procedure until there are no such two elements of Thick $\left(\boldsymbol{G}^{\prime}\right)$ and $\operatorname{Thin}\left(\boldsymbol{G}^{\prime}\right)$, then we get the resulting GHS $\boldsymbol{G}$ of $M$ from the GHS $\boldsymbol{G}^{\prime}$ [2, Lemma 5.4], and we say $\boldsymbol{G}$ is obtained from $\boldsymbol{G}^{\prime}$ by cleaning. We say the GHS $\boldsymbol{G}$ of $M$ given by preweak reduction along $(V, W)$, followed by cleaning, is obtained from $\boldsymbol{H}$ by weak reduction along $(V, W)$.

Definition 2.12 (Kim [13, Definition 2.12]) In a weak reducing pair for a Heegaard splitting $(\mathcal{V}, \mathcal{W} ; F)$, if a disk belongs to $\mathcal{V}$, then we call it a $\mathcal{V}$-disk. Otherwise, we call it a $\mathcal{W}$-disk. We call a 2-simplex in $\mathcal{D}_{\mathcal{V W}}(F)$ represented by two vertices in $\mathcal{D}_{\mathcal{V}}(F)$ and one vertex in $\mathcal{D}_{\mathcal{W}}(F)$ a $\mathcal{V}$-face, and likewise define a $\mathcal{W}$-face. Let us consider a 1-dimensional graph as follows:

(1) We assign a vertex to each $\mathcal{V}$-face in $\mathcal{D}_{\mathcal{V W}}(F)$.

(2) If a $\mathcal{V}$-face shares a weak reducing pair with another $\mathcal{V}$-face, then we assign an edge between these two vertices in the graph. 
We call this graph the graph of $\mathcal{V}$-faces. If there is a maximal subset $\varepsilon \mathcal{V}$ of $\mathcal{V}$-faces in $\mathcal{D}_{\mathcal{V W}}(F)$ representing a connected component of the graph of $\mathcal{V}$-faces and the component is not an isolated vertex, then we call $\varepsilon \mathcal{V}$ a $\mathcal{V}$-facial cluster. Similarly, we define the graph of $\mathcal{W}$-faces and a $\mathcal{W}$-facial cluster. In a $\mathcal{V}$-facial cluster, every weak reducing pair gives the common $\mathcal{W}$-disk, and vice versa.

If we consider an unstabilized genus-three Heegaard splitting of an irreducible manifold, we obtain the following lemmas.

Lemma 2.13 (Kim [13, Lemma 2.13]) Suppose that $M$ is an irreducible 3-manifold and $(\mathcal{V}, \mathcal{W} ; F)$ is an unstabilized genus-three Heegaard splitting of $M$. If there are two $\mathcal{V}$-faces $f_{1}$ represented by $\left\{V_{0}, V_{1}, W\right\}$ and $f_{2}$ represented by $\left\{V_{1}, V_{2}, W\right\}$ sharing a weak reducing pair $\left(V_{1}, W\right)$, then $\partial V_{1}$ is nonseparating, and $\partial V_{0}, \partial V_{2}$ are separating in $F$. Therefore, there is a unique weak reducing pair in a $\mathcal{V}$-facial cluster which can belong to two or more faces in the $\mathcal{V}$-facial cluster.

Definition 2.14 (Kim [13, Definition 2.14]) By Lemma 2.13, there is a unique weak reducing pair in a $\mathcal{V}$-facial cluster belonging to two or more faces in the cluster. We call it the center of the cluster, and call the other weak reducing pairs hands of the cluster; see Figure 2.

Lemma 2.15 (Kim [13, Lemma 2.15]) Let $M$ and $F$ be as in Lemma 2.13. Every $\mathcal{V}-$ face belongs to some $\mathcal{V}$-facial cluster. Moreover, every $\mathcal{V}$-facial cluster has infinitely many hands.

Note that if a $\mathcal{V}$-face is represented by two weak reducing pairs, then one is the center and the other is a hand. Lemma 2.13 means that the $\mathcal{V}$-disk in the center of a $\mathcal{V}$-facial cluster is nonseparating, and those from hands are all separating. Moreover, Lemma 2.9 implies that the $\mathcal{V}$-disk of a hand of a $\mathcal{V}$-facial cluster (i) is a band-sum of two parallel copies of the $\mathcal{V}$-disk of the center of the cluster, and (ii) determines the $\mathcal{V}$-disk of the center by the uniqueness of the meridian disk of the solid torus cut off from $\mathcal{V}$ by the $\mathcal{V}$-disk of the hand.

Note also that every $\mathcal{V}$ - or $\mathcal{W}$-facial cluster is contractible (see Figure 2).

The following lemma means that the isotopy class of the generalized Heegaard splitting obtained by weak reduction along a weak reducing pair does not depend on the choice of the weak reducing pair if the weak reducing pair varies in a fixed $\mathcal{V}-$ or $\mathcal{W}$-facial cluster. 


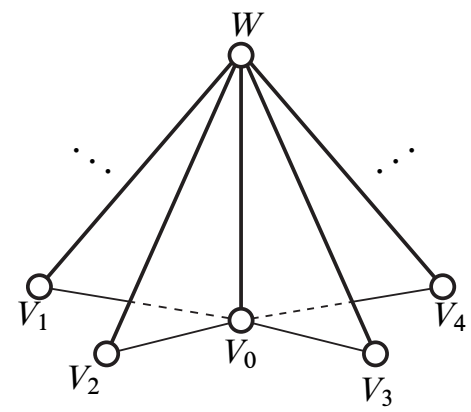

Figure 2: An example of a $\mathcal{V}$-facial cluster in $\mathcal{D}_{\mathcal{V W}}(F) .\left(V_{0}, W\right)$ is the center and the other weak reducing pairs are hands.

Lemma 2.16 Let $M$ and $F$ be as in Lemma 2.13. Every weak reducing pair in a $\mathcal{V}$-face gives the same generalized Heegaard splitting after weak reduction up to isotopy. Therefore, every weak reducing pair in a $\mathcal{V}$-facial cluster gives the same generalized Heegaard splitting after weak reduction up to isotopy. Moreover, the embedding of the thick level contained in $\mathcal{V}$ or $\mathcal{W}$ does not vary in the relevant compression body up to isotopy.

Proof Let $(V, W)$ be the center of a $\mathcal{V}$-facial cluster and $\left(V^{\prime}, W\right)$ be a hand of the $\mathcal{V}$-facial cluster. Here, $V$ is nonseparating while $V^{\prime}$ is separating in $\mathcal{V}$ by Lemma 2.13. Let $\boldsymbol{H}$ and $\boldsymbol{H}^{\prime}$ be the generalized Heegaard splittings obtained by weak reductions along $(V, W)$ and $\left(V^{\prime}, W\right)$ from $(\mathcal{V}, \mathcal{W} ; F)$, respectively. It is sufficient to show that $\boldsymbol{H}$ and $\boldsymbol{H}^{\prime}$ are the same up to isotopy.

Claim Both $\overline{\operatorname{Thin}}(\boldsymbol{H})$ and $\overline{\operatorname{Thin}}\left(\boldsymbol{H}^{\prime}\right)$ consist of one component.

Proof of claim Suppose that $\overline{\operatorname{Thin}}(\boldsymbol{H})$ or $\overline{\operatorname{Thin}}\left(\boldsymbol{H}^{\prime}\right)$ does not consist of one component. We claim that each component of the inner thin level of the generalized Heegaard splitting obtained by weak reduction must have scars of both disks of the weak reducing pair. Let us consider an arbitrary weak reducing pair $(D, E)$. Then $\partial E$ must belong to the genus-two component of $F-\partial D$ by Lemma 2.8 and vice versa. Hence, we get the following in the GHS obtained by the preweak reduction along $(D, E)$ :

(1) $F_{D E}$ has a component without scars of both $D$ and $E$ if and only if at least one of $D$ or $E$ is separating.

(2) If one of $D$ and $E$ is separating, say $D$, then we can find a product region in the GHS cobounded by the isotoped genus-one component of $F_{D}$ into the interior of $\mathcal{V}$ and the torus component of $F_{D E}$ with only the scar of $D$.

Therefore, every component of $F_{D E}$ without scars of both $D$ and $E$ disappears after cleaning; see Figure 3 or Figure 5. 


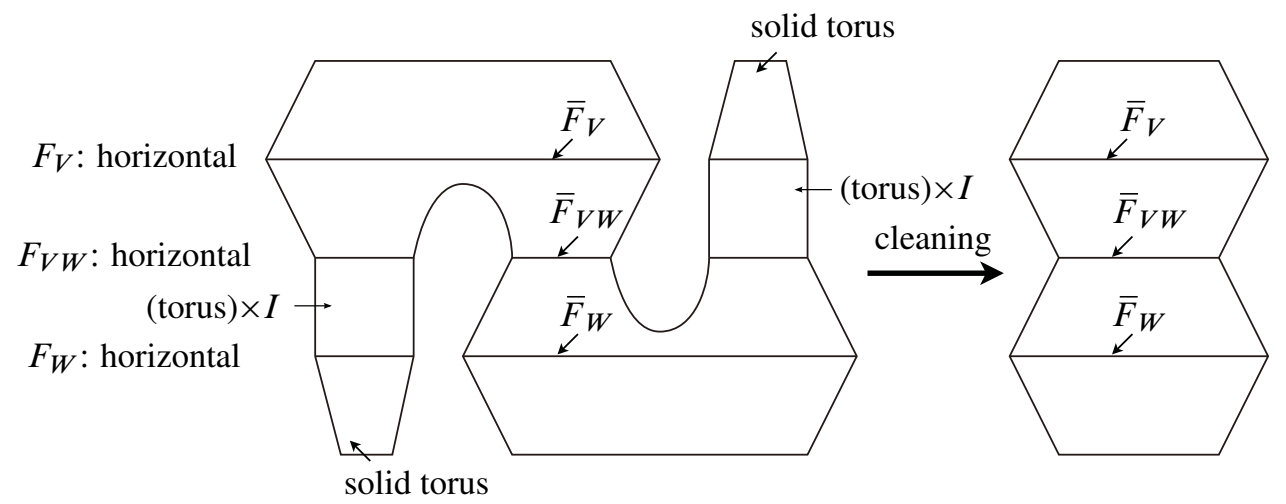

Figure 3: The inner thin level comes from the component of $F_{V W}$ with scars of both $V$ and $W$ after cleaning.

Hence, if we try all possible weak reductions by considering Lemma 2.8 (see the Appendix), then the only case for disconnected inner thin level is when both disks are nonseparating but the union of boundaries of them is separating in $F$, that is, the inner thin level consists of two tori (see Figure 4). This means that $\overline{\operatorname{Thin}}\left(\boldsymbol{H}^{\prime}\right)$ must consist of only one component since $V^{\prime}$ is separating. Hence, $\overline{\operatorname{Thin}}(\boldsymbol{H})$ is disconnected, that is, $W$ is nonseparating and $\partial V \cup \partial W$ is separating in $F$. But Lemma 2.9 forces $V^{\prime}$ to be a band-sum of two parallel copies of $V$. Here, $V^{\prime}$ must intersect $W$ otherwise we can find an arc in $F$ realizing the band-sum but missing $\partial W$, that is, $\partial V \cup \partial W$ is nonseparating, violating the assumption that $\partial V \cup \partial W$ is separating in $F$. But this violates the assumption that $\left(V^{\prime}, W\right)$ is a weak reducing pair.

This completes the proof of the claim.

Recall that given a GHS $\boldsymbol{G}^{\prime}$ obtained by preweak reduction along a weak reducing pair $(D, E)$, the isotoped genus-one component of $F_{D}$ into $\mathcal{V}$ in Thick $\left(\boldsymbol{G}^{\prime}\right)$ disappears after cleaning when $D$ is separating, as we have seen in the proof of the claim and the symmetric argument also holds for the isotoped genus-one component of $F_{E}$ into $\mathcal{W}$ in $\operatorname{Thick}\left(\boldsymbol{G}^{\prime}\right)$ when $E$ is separating.

Hence, considering Thick $(\boldsymbol{H})$ and $\overline{\operatorname{Thin}}(\boldsymbol{H})$,

$$
\operatorname{Thick}(\boldsymbol{H})=\left\{F_{V}, \bar{F}_{W}\right\} \text { and } \overline{\operatorname{Thin}}(\boldsymbol{H})=\left\{\bar{F}_{V W}\right\},
$$

where $\bar{F}_{W}$ comes from the genus-two component of $F_{W}$ and $\bar{F}_{V W}$ is the component of $F_{V W}$ with scars of both $V$ and $W$ (if there is no confusion, then we will use the terms $F_{V}$ or $\bar{F}_{V}$ as the component isotoped into the interior of $\mathcal{V}$ for the cases of thick levels). 
Similarly, for Thick $\left(\boldsymbol{H}^{\prime}\right)$ and $\operatorname{Thin}\left(\boldsymbol{H}^{\prime}\right)$,

$$
\operatorname{Thick}\left(\boldsymbol{H}^{\prime}\right)=\left\{\bar{F}_{V^{\prime}}, \bar{F}_{W}\right\} \text { and } \overline{\operatorname{Thin}}\left(\boldsymbol{H}^{\prime}\right)=\left\{\bar{F}_{V^{\prime} W}\right\},
$$

where $\bar{F}_{V^{\prime}}$ comes from the genus-two component of $F_{V^{\prime}}$ and $\bar{F}_{V^{\prime} W}$ is the component of $F_{V^{\prime} W}$ with scars of both $V^{\prime}$ and $W$. Here, $V^{\prime}$ must cut off a solid torus $\mathcal{V}^{\prime}$ from $\mathcal{V}$ and $V$ is a meridian disk of $\mathcal{V}^{\prime}$ by Lemma 2.9, that is, $F_{V}$ is isotopic to $\bar{F}_{V^{\prime}}$ in $\mathcal{V}$, so it is in $M$. Moreover, $\bar{F}_{V W}$ is isotopic to $\bar{F}_{V^{\prime} W}$ similarly, since $\partial W$ must belong to the genus-two component of $F-\partial V^{\prime}$ by Lemma 2.8. Hence, Thick $(\boldsymbol{H})=\operatorname{Thick}\left(\boldsymbol{H}^{\prime}\right)$ and Thin $(\boldsymbol{H})=$ Thin $\left(\boldsymbol{H}^{\prime}\right)$ up to isotopy and indeed we can naturally imagine the ambient isotopy moving $\boldsymbol{H}$ into $\boldsymbol{H}^{\prime}$. This completes the first and the second statements.

The last statement has already been proven in the previous paragraph. This completes the proof of Lemma 2.16.

The next lemma gives an upper bound for the dimension of $\mathcal{D}_{\mathcal{V W}}(F)$ and restricts the shape of a 3-simplex in $\mathcal{D}_{\mathcal{V W}}(F)$.

Lemma 2.17 (Kim [12, Proposition 2.10]) Let $M$ and $F$ be as in Lemma 2.13. Then $\operatorname{dim}\left(\mathcal{D}_{\mathcal{V W}}(F)\right) \leq 3$. Moreover, if $\operatorname{dim}\left(\mathcal{D}_{\mathcal{V W}}(F)\right)=3$, then every 3-simplex in $\mathcal{D}_{\mathcal{V W}}(F)$ must have the form $\left\{V_{1}, V_{2}, W_{1}, W_{2}\right\}$, where $V_{1}, V_{2} \subset \mathcal{V}$ and $W_{1}, W_{2} \subset \mathcal{W}$. Indeed, $V_{1}\left(\operatorname{resp} W_{1}\right)$ is nonseparating in $\mathcal{V}(\operatorname{resp}$ in $\mathcal{W})$ and $V_{2}\left(\operatorname{resp} W_{2}\right)$ is a bandsum of two parallel copies of $V_{1}$ in $\mathcal{V}\left(\operatorname{resp} W_{1}\right.$ in $\left.\mathcal{W}\right)$.

Note that the third statement of Lemma 2.17 is obtained by applying Lemma 2.9 to the $\mathcal{V}$-face $\left\{V_{1}, V_{2}, W_{1}\right\}$ and the $\mathcal{W}$-face $\left\{V_{2}, W_{1}, W_{2}\right\}$.

The next lemma gives natural but important observations for genus $g \geq 2$ compression bodies containing a negative boundary component of genus $g-1$. The proof comes from a standard outermost disk argument for the intersection of two compressing disks in $\mathcal{V}$ when we consider the uniqueness of the desired disk. We can find a rigorous proof in Ido, Jang and Kobayashi [11, Lemma 3.3].

Lemma 2.18 Let $\mathcal{V}$ be a genus $g \geq 2$ compression body with $\partial_{-} \mathcal{V}$ containing a genus $g-1$ surface. Then the following hold:

(1) If $\partial_{-} \mathcal{V}$ is connected (that is, $\partial_{-} \mathcal{V}$ consists of a genus $g-1$ surface), then there is a unique nonseparating disk in $\mathcal{V}$ up to isotopy.

(2) If $\partial_{-} \mathcal{V}$ is disconnected (that is, $\partial_{-} \mathcal{V}$ consists of a genus $g-1$ surface and a torus), then there is a unique compressing disk in $\mathcal{V}$ up to isotopy and it is a separating disk. 


\section{The proof of Theorem 1.1}

Lemma 3.1 Suppose that $M$ is an orientable, irreducible 3-manifold and $(\mathcal{V}, \mathcal{W} ; F)$ is an unstabilized genus-three Heegaard splitting of $M$. If there exist two weak reducing pairs such that the generalized Heegaard splittings obtained by weak reductions along these weak reducing pairs are not isotopic in $M$, then $F$ is critical.

Before proving Lemma 3.1, we introduce the notion of distance between two weak reducing pairs.

Definition 3.2 (Bachman [2, Definition 8.3]) Suppose $F$ is a Heegaard surface in a 3-manifold. Let $\left(V_{i}, W_{i}\right)$ be a weak reducing pair for $F$ for $i=0,1$. Then we define the distance between $\left(V_{0}, W_{0}\right)$ and $\left(V_{1}, W_{1}\right)$ to be the smallest $n$ for which there is a sequence $\left\{D_{j}\right\}_{j=0}^{n+1}$ such that

(1) $\left\{D_{0}, D_{1}\right\}=\left\{V_{0}, W_{0}\right\}$,

(2) $\left\{D_{n}, D_{n+1}\right\}=\left\{V_{1}, W_{1}\right\}$,

(3) for all $j$ the pair $\left\{D_{j}, D_{j+1}\right\}$ gives a weak reducing pair for $F$,

(4) for $1 \leq j \leq n, D_{j-1}$ is disjoint from, or equal to, $D_{j+1}$.

If there is no such sequence, then we define the distance to be $\infty$.

Lemma 3.3 (Bachman [2, Lemma 8.5]) Suppose $F$ is a Heegaard surface in a 3manifold. If there are weak reducing pairs $(V, W)$ and $\left(V^{\prime}, W^{\prime}\right)$ for $F$ such that the distance between them is $\infty$, then $F$ is critical.

Proof of Lemma 3.1 Suppose that there are two weak reducing pairs $(V, W)$ and $\left(V^{\prime}, W^{\prime}\right)$ for $(\mathcal{V}, \mathcal{W} ; F)$ such that the generalized Heegaard splittings $\boldsymbol{H}$ and $\boldsymbol{H}^{\prime}$ obtained by weak reductions along these pairs are not isotopic in $M$.

If the distance between $(V, W)$ and $\left(V^{\prime}, W^{\prime}\right)$ is $\infty$, then $F$ is critical by Lemma 3.3. Assume that the distance between $(V, W)$ and $\left(V^{\prime}, W^{\prime}\right)$ is $k<\infty$. If $k \leq 1$, then either $(V, W)=\left(V^{\prime}, W^{\prime}\right)$ or they are contained in a $\mathcal{V}$ - or $\mathcal{W}$-face. This leads to a contradiction by Lemma 2.16. Hence, assume that $k \geq 2$. Let $\left\{D_{0}, D_{1}, \ldots, D_{k}, D_{k+1}\right\}$ be the sequence of compressing disks of $F$ realizing the distance between $(V, W)$ and $\left(V^{\prime}, W^{\prime}\right)$, where $\left\{D_{0}, D_{1}\right\}=\{V, W\}$ and $\left\{D_{k}, D_{k+1}\right\}=\left\{V^{\prime}, W^{\prime}\right\}$. Reading the above sequence from left to right, we get a sequence of $\mathcal{V}$ - and $\mathcal{W}$-faces $\Delta_{0}, \ldots, \Delta_{n}$ such that

(1) $(V, W) \subset \Delta_{0}$ and $\left(V^{\prime}, W^{\prime}\right) \subset \Delta_{n}$,

(2) $\Delta_{i-1}$ shares a weak reducing pair with $\Delta_{i}$ for $i=1, \ldots, n$. 


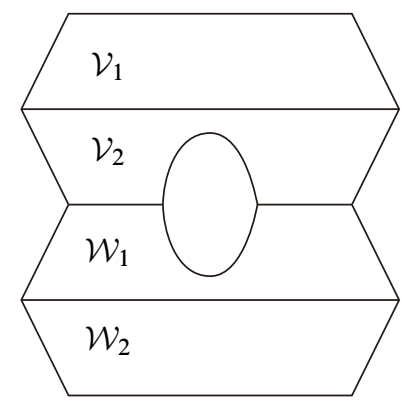

Figure 4: The inner thin level consists of two tori.

If we consider the generalized Heegaard splitting corresponding to $\Delta_{i}$ inductively from $i=0$ to $n$ using Lemma 2.16 and the assumption that $\Delta_{i-1}$ shares a weak reducing pair with $\Delta_{i}$, then we see that $\boldsymbol{H}$ and $\boldsymbol{H}^{\prime}$ are isotopic, violating the assumption. Hence, the distance between $(V, W)$ and $\left(V^{\prime}, W^{\prime}\right)$ cannot be finite. This completes the proof.

Let $(\mathcal{V}, \mathcal{W} ; F)$ be a weakly reducible unstabilized genus-three Heegaard splitting of an irreducible 3-manifold $M$. Considering Lemma 3.1, we assume that the generalized Heegaard splitting obtained by weak reduction from $(\mathcal{V}, \mathcal{W} ; F)$ is unique up to isotopy. Considering the generalized Heegaard splitting obtained by weak reduction along $(V, W)$ from $(\mathcal{V}, \mathcal{W} ; F)$, the inner thin level would consist of a torus or two tori, where the latter case holds only when both disks of the weak reducing pair are nonseparating but the union of the boundaries is separating in $F$, as we have checked in the proof of Lemma 2.16; see Figure 4. The thick levels would come from the genus-two components of $F_{V}$ and $F_{W}$, say $\bar{F}_{V}$ and $\bar{F}_{W}$, as we have checked in the proof of Lemma 2.16. But even though we assumed that the generalized Heegaard splitting obtained by weak reduction from $(\mathcal{V}, \mathcal{W} ; F)$ is unique up to isotopy, it is not clear that both $\bar{F}_{V}$ and $\bar{F}_{W}$ should be unique up to isotopy in $\mathcal{V}$ and $\mathcal{W}$, respectively, not in the entire $M$. Indeed, we can imagine an ambient isotopy $f_{t}$ defined on $M$ such that $f_{0}$ is the identity map on $M, f_{1}\left(\bar{F}_{V}\right) \cap \mathcal{W}=\varnothing, \bar{F}_{V}$ is not isotopic to $f_{1}\left(\bar{F}_{V}\right)$ in $\mathcal{V}$, and $f_{t}\left(\bar{F}_{V}\right) \cap \mathcal{W} \neq \varnothing$ for some $t$. If the isotoped generalized Heegaard splitting itself is also that obtained by weak reduction from $(\mathcal{V}, \mathcal{W} ; F)$, then there would be another weak reducing pair $\left(V^{\prime}, W^{\prime}\right)$ for $(\mathcal{V}, \mathcal{W} ; F)$ such that $f_{1}\left(\bar{F}_{V}\right)$ is isotopic to the one obtained by pushing the genus-two component of $F_{V^{\prime}}$ off into the interior of $\mathcal{V}$ in $\mathcal{V}$ but $V$ is not isotopic to $V^{\prime}$ in $\mathcal{V}$. Hence, we need the following lemma.

Lemma 3.4 Let $M$ and $F$ be as in Lemma 3.1. Suppose that there are two generalized Heegaard splittings $\boldsymbol{H}_{1}$ and $\boldsymbol{H}_{2}$ obtained by weak reductions along $\left(V_{1}, W_{1}\right)$ and $\left(V_{2}, W_{2}\right)$ from $(\mathcal{V}, \mathcal{W} ; F)$, respectively, such that the thick levels of $\boldsymbol{H}_{1}$ and $\boldsymbol{H}_{2}$ embedded in the interior of $\mathcal{V}$ are nonisotopic in $\mathcal{V}$. (It may be possible that $\boldsymbol{H}_{1}$ is the same as $\boldsymbol{H}_{2}$ in $M$ up to isotopy.) Then $F$ is critical. 

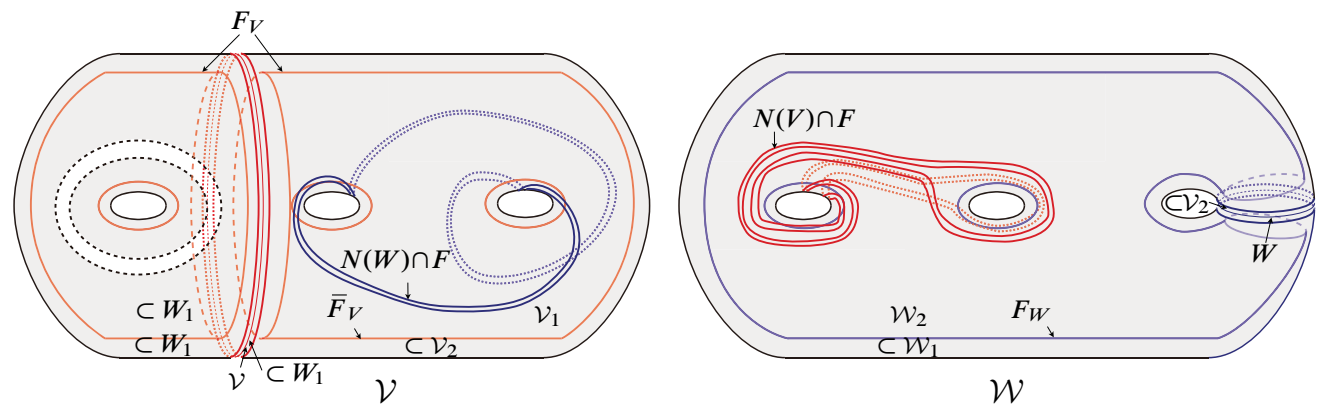

Figure 5: $\mathcal{W}_{1} \cap \mathcal{W} \cong \bar{F}_{W} \times I$ and $\mathcal{V}_{2} \cap \mathcal{V} \cong \bar{F}_{V} \times I$

Proof Say $\boldsymbol{H}_{i}=\left(\mathcal{V}_{1}^{i}, \mathcal{V}_{2}^{i} ; \bar{F}_{V_{i}}\right) \cup\left(\mathcal{W}_{1}^{i}, \mathcal{W}_{2}^{i} ; \bar{F}_{W_{i}}\right)$, where $\partial_{-} \mathcal{V}_{2}^{i} \cap \partial_{-} \mathcal{W}_{1}^{i} \neq \varnothing$ and let $\mathcal{V}_{i}^{\prime}$ be the region between $\bar{F}_{V_{i}}$ and $\partial_{+} \mathcal{V}$ in $\mathcal{V}$ for $i=1,2$. We can see that $\mathcal{V}_{i}^{\prime}$ is a genus-three compression body, where either $\partial_{-} \mathcal{V}_{i}^{\prime}$ consists of (i) a genus-two surface if $\partial_{-} \mathcal{V}_{i}^{\prime}$ is connected or (ii) a torus and a genus-two surface if $\partial_{-} \mathcal{V}_{i}^{\prime}$ is disconnected for $i=1,2$, and $V_{i}$ is a compressing disk of $\mathcal{V}_{i}^{\prime}$ for $i=1,2$ by construction.

Claim If one of $\partial_{-} \mathcal{V}_{1}^{\prime}$ and $\partial_{-} \mathcal{V}_{2}^{\prime}$ is connected and the other is disconnected, then $F$ is critical.

Proof of claim Suppose that $\partial_{-} \mathcal{V}_{1}^{\prime}$ is connected but $\partial_{-} \mathcal{V}_{2}^{\prime}$ is disconnected.

Considering $V_{1}$, we see that either $V_{1}$ is nonseparating in $\mathcal{V}$, or $V_{1}$ cuts off a solid torus from $\mathcal{V}$ since $\partial_{-} \mathcal{V}_{1}^{\prime}$ is connected. If $\partial_{-} \mathcal{W}_{1}^{1}$ has a component not belonging to the inner thin level, then this component cannot come from $\partial_{-} \mathcal{W}$ since $\mathcal{W}_{1}^{1} \cap \mathcal{W}$ is the region in $\mathcal{W}$ between the thick level $\bar{F}_{W_{1}}$ and the genus-two component of $F_{W_{1}}$ which is homeomorphic to $\bar{F}_{W_{1}} \times I$; see Figure 5. Hence, this component comes from $\partial_{-} \mathcal{V}$ after cleaning (see Figure 6(b) or (c), where $\partial_{-} \mathcal{V}$ comes from the top horizontal line) and therefore $V_{1}$ must cut off (torus) $\times I$ from $\mathcal{V}$, violating the assumption. Therefore, $\partial_{-} \mathcal{W}_{1}^{1}$ is the inner thin level itself, that is, $\partial_{-} \mathcal{W}_{1}^{1} \cap \partial_{-} \mathcal{V}=\varnothing$

Now we consider $V_{2}$. Since $\mathcal{V}_{2}^{\prime}$ is a genus-three compression body such that $\partial_{-} \mathcal{V}_{2}^{\prime}$ consists of a torus and a genus-two surface, $V_{2}$ must cut off (torus) $\times I$ from $\mathcal{V}$ by Lemma 2.18. Hence, the region cut by a copy of $V_{2}$ from $\mathcal{V}$ which is homeomorphic to (torus) $\times I$ would be attached to the product region in $\mathcal{W}$ between the thick level $\bar{F}_{W_{2}}$ and the genus-two component of $F_{W_{2}}$ to complete $\mathcal{W}_{1}^{2}$; see Figure 5 or Figure 6(b) or (c). That is, $\partial_{-} \mathcal{W}_{1}^{2} \cap \partial_{-} \mathcal{V} \neq \varnothing$.

This means that if $\boldsymbol{H}_{1}$ is isotopic to $\boldsymbol{H}_{2}$, then the isotopy cannot take $\mathcal{W}_{1}^{1}$ into $\mathcal{W}_{1}^{2}$, that is, it takes $\mathcal{V}_{2}^{1}$ into $\mathcal{W}_{1}^{2}$ since the isotopy takes the inner thin level of $\boldsymbol{H}_{1}$ into that of $\boldsymbol{H}_{2}$. But $\partial_{-} \mathcal{V}_{2}^{1} \cap \partial M \subset \partial_{-} \mathcal{W}$ if it is nonempty (for example, we can refer 
to Figure 6(c)). Since the isotopy cannot change $\partial_{-} \mathcal{V}_{2}^{1} \cap \partial M$, we conclude that $\boldsymbol{H}_{1}$ cannot be isotopic to $\boldsymbol{H}_{2}$ in $M$. Therefore, $F$ is critical by Lemma 3.1. This completes the proof of the claim.

By the claim, we can assume that both $\partial_{-} \mathcal{V}_{1}^{\prime}$ and $\partial_{-} \mathcal{V}_{2}^{\prime}$ are connected or both $\partial_{-} \mathcal{V}_{1}^{\prime}$ and $\partial_{-} \mathcal{V}_{2}^{\prime}$ are disconnected. If $V_{1}$ is isotopic to $V_{2}$ in $\mathcal{V}$, then $\bar{F}_{V_{1}}$ would be isotopic to $\bar{F}_{V_{2}}$ in $\mathcal{V}$ in any case, violating the assumption. Hence, $V_{1}$ is not isotopic to $V_{2}$ in $\mathcal{V}$.

Suppose that both $\partial_{-} \mathcal{V}_{1}^{\prime}$ and $\partial_{-} \mathcal{V}_{2}^{\prime}$ are connected. If $V_{i}$ is separating in $\mathcal{V}_{i}^{\prime}$, then it must cut off a solid torus from $\mathcal{V}_{i}^{\prime}$ since $\mathcal{V}_{i}^{\prime}$ is a genus-three compression body such that $\partial_{-} \mathcal{V}_{i}^{\prime}$ consists of a genus-two surface. Hence, we can take a meridian disk $V_{i}^{\prime}$ of the solid torus that $V_{i}$ cuts off from $\mathcal{V}_{i}^{\prime}$ so that it would miss $V_{i}$. Moreover, $V_{i}^{\prime} \cap W_{i}=\varnothing$ by Lemma 2.8. That is, we get the $\mathcal{V}$-face $\left\{V_{i}^{\prime}, V_{i}, W_{i}\right\}$. Hence, we can assume that $V_{i}$ is nonseparating without changing the isotopy class of $\boldsymbol{H}_{i}$ and the embedding of $\bar{F}_{V_{i}}$ in $\mathcal{V}$ up to isotopy by Lemma 2.16. Since $V_{1}$ is not isotopic to $V_{2}$ in $\mathcal{V}$ and both disks are nonseparating in $\mathcal{V}, F$ is critical by $\operatorname{Kim}$ [12, Theorem 1.1].

Hence, we can assume that both $\partial_{-} \mathcal{V}_{1}^{\prime}$ and $\partial_{-} \mathcal{V}_{2}^{\prime}$ are disconnected, that is, each $V_{i}$ cuts off (torus) $\times I$ from $\mathcal{V}_{i}^{\prime}$, so also does in $\mathcal{V}$, for $i=1,2$. We claim that the distance defined in Definition 3.2 between $\left(V_{1}, W_{1}\right)$ and $\left(V_{2}, W_{2}\right)$ is $\infty$.

For the sake of contradiction, assume that the distance is finite. Then we get a sequence of $\mathcal{V}$ - and $\mathcal{W}$-faces $\Delta_{0}, \ldots, \Delta_{n}$ such that

(1) $\left(V_{1}, W_{1}\right) \subset \Delta_{0}$ and $\left(V_{2}, W_{2}\right) \subset \Delta_{n}$,

(2) $\Delta_{i-1}$ shares a weak reducing pair with $\Delta_{i}$ for $i=1, \ldots, n$,

similar to the proof of Lemma 3.1. Since $V_{1}$ is not isotopic to $V_{2}$ in $\mathcal{V}$, there must be a $\mathcal{V}$-face among $\Delta_{0}, \ldots, \Delta_{n}$. Let $\Delta_{k}$ be the first $\mathcal{V}$-face, that is, it contains $V_{1}$. Here, every $\mathcal{V}$-face contains a nonseparating $\mathcal{V}$-disk and the boundary of it must be a nonseparating loop in the punctured torus that the boundary of the separating $\mathcal{V}$-disk in the $\mathcal{V}$-face cuts off from $F$ by Lemma 2.9. But the condition that $V_{1}$ cuts off (torus) $\times I$ from $\mathcal{V}$ means that there cannot be such a nonseparating $\mathcal{V}$-disk in $\Delta_{k}$, leading to a contradiction.

Hence, the distance between $\left(V_{1}, W_{1}\right)$ and $\left(V_{2}, W_{2}\right)$ is $\infty$, that is, $F$ is critical by Lemma 3.3. This completes the proof of Lemma 3.4.

The next lemma deals with the case where the inner thin level consists of a torus. 
Lemma 3.5 Let $(\mathcal{V}, \mathcal{W} ; F)$ be a weakly reducible unstabilized genus-three Heegaard splitting in an orientable irreducible 3-manifold. If every weak reducing pair of $F$ gives the same generalized Heegaard splitting obtained by weak reduction up to isotopy such that the inner thin level consists of a torus and the embedding of each thick level in the relevant compression body is unique up to isotopy, then $\mathcal{D}(F)$ is contractible.

Proof Let $(V, W)$ be a weak reducing pair for $(\mathcal{V}, \mathcal{W} ; F)$ and $T$ the inner thin level of the generalized Heegaard splitting obtained by weak reduction.

If one of $V$ or $W$, say $V$, cuts off a solid torus from $\mathcal{V}$, then $\partial W$ cannot belong to the once-punctured torus that $\partial V$ cuts off from $F$ by Lemma 2.8. Hence, we can take a nonseparating disk $V^{\prime}$ from the solid torus and we can assume that $V^{\prime} \cap(V \cup W)=\varnothing$. Of course, the generalized Heegaard splitting obtained by weak reduction along $(V, W)$ is the same as the one obtained along $\left(V^{\prime}, W\right)$ and the embeddings of thick levels in the relevant compression bodies are the same up to isotopy by Lemma 2.16. Hence, without loss of generality, there are three types of the generalized Heegaard splittings obtained by weak reductions as follows, where these cases come from the shape of the two compression bodies sharing the inner thin level.

Case (a) $\quad V$ and $W$ are nonseparating in $\mathcal{V}$ and $\mathcal{W}$, respectively, that is, the negative boundaries of the two compression bodies are connected (see Figure 6(a)).

Case (b) $V$ cuts off (torus) $\times I$ from $\mathcal{V}$ and $W$ is nonseparating in $\mathcal{W}$, that is, the negative boundary of one compression body is connected but that of the other is disconnected (see Figure 6(b)).

Case (c) $V$ and $W$ each cut off (torus) $\times I$ from $\mathcal{V}$ and $\mathcal{W}$, respectively, that is, the negative boundaries of the two compression bodies are disconnected (see Figure 6(c)).

These three cases are mutually exclusive by the assumption that the generalized Heegaard splitting obtained by weak reduction is unique up to isotopy. Note that there is the symmetric case for Case (b) when $W$ cuts off (torus) $\times I$ from $\mathcal{W}$, but the shape of the generalized Heegaard splitting is just the one obtained by turning the figure upside down.

In Case (a), $\partial V \cup \partial W$ is nonseparating in $F$ since the inner thin level consists of a torus.

Let us consider the generalized Heegaard splitting obtained by weak reduction along $(V, W)$. Here, we can see that the preweak reduction along $(V, W)$ is exactly the same as the weak reduction along $(V, W)$. Hence, it consists of two splittings $\left(\mathcal{V}_{1}, \mathcal{V}_{2} ; F_{V}\right)$ and $\left(\mathcal{W}_{1}, \mathcal{W}_{2} ; F_{W}\right)$ such that $\partial_{-} \mathcal{V}_{2}=\partial_{-} \mathcal{W}_{1}=T$. Let $\mathcal{V}^{\prime}$ (resp $\left.\mathcal{W}^{\prime}\right)$ be the region 


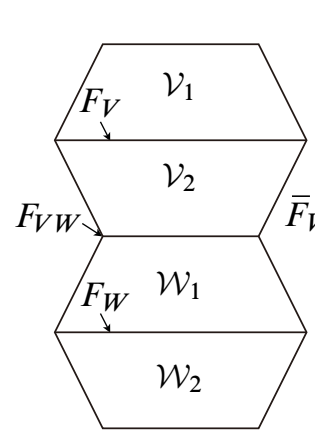

(a)

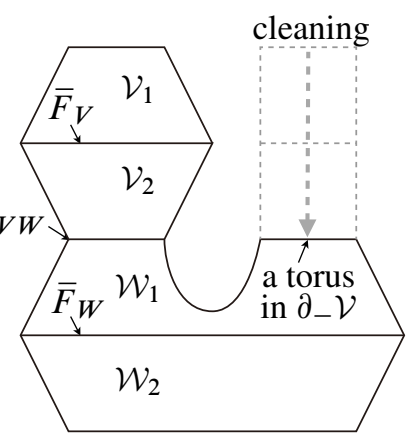

(b)

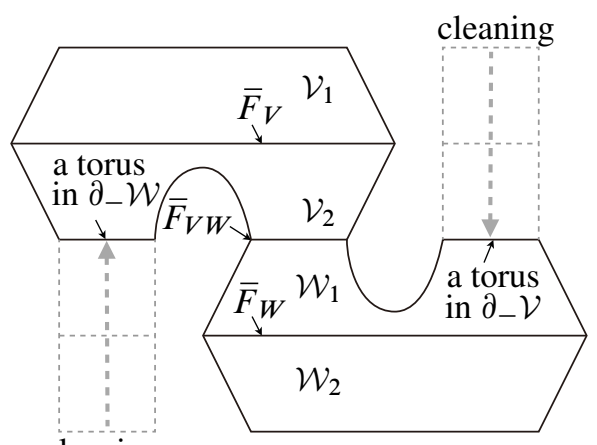

(c)

Figure 6: The GHSs for the three cases.

between $\partial_{+} \mathcal{V}$ and $F_{V}$ in $\mathcal{V}$ (resp $\partial_{+} \mathcal{W}$ and $F_{W}$ in $\mathcal{W}$ ). Then we can see that $V$ and $W$ are nonseparating compressing disks of $\mathcal{V}^{\prime}$ and $\mathcal{W}^{\prime}$, respectively. Since $\mathcal{V}^{\prime}$ and $\mathcal{W}^{\prime}$ are genus-three compression bodies with negative boundary consisting of a genus-two surface, $V$ and $W$ are uniquely determined in $\mathcal{V}^{\prime}$ and $\mathcal{W}^{\prime}$, respectively, up to isotopy. Hence, we get the induced isotopy classes of $V$ and $W$ in $\mathcal{V}$ and $\mathcal{W}$ from the isotopy classes of $V$ and $W$ in $\mathcal{V}^{\prime}$ and $\mathcal{W}^{\prime}$, respectively. Moreover, the uniqueness of the isotopy classes of the embeddings of the thick levels of the generalized Heegaard splitting obtained by weak reduction from $(\mathcal{V}, \mathcal{W} ; F)$ in the relevant compression bodies forces the choice of the induced isotopy classes of $V$ and $W$ in $\mathcal{V}$ and $\mathcal{W}$ to be unique. This means that we can consider the weak reducing pair $(V, W)$ as the fixed pair $(\bar{V}, \bar{W})$ even though we've chosen an arbitrary weak reducing pair consisting of nonseparating disks.

Let us consider an arbitrary weak reducing pair $\left(V^{*}, W^{*}\right)$ for $(\mathcal{V}, \mathcal{W} ; F)$.

If both $V^{*}$ and $W^{*}$ are nonseparating, then $\left(V^{*}, W^{*}\right)$ must be $(\bar{V}, \bar{W})$ by the previous argument, and therefore $\partial V^{*} \cup \partial W^{*}$ is nonseparating in $F$. This means that we can take a band-sum of two parallel copies of $V^{*}$ in $\mathcal{V}$, say $V^{\prime}$, and a band-sum of two parallel copies of $W^{*}$ in $\mathcal{W}$, say $W^{\prime}$, such that $\left\{V^{\prime}, V^{*}=\bar{V}, W^{*}=\bar{W}, W^{\prime}\right\}$ forms a 3-simplex.

If exactly one of $V^{*}$ and $W^{*}$ is nonseparating, say $V^{*}$, then $W^{*}$ must cut off a solid torus from $\mathcal{W}$ (otherwise, $W^{*}$ cuts off (torus) $\times I$ from $\mathcal{W}$ and therefore the generalized Heegaard splitting obtained by weak reduction along $\left(V^{*}, W^{*}\right)$ would be the symmetric case of Case (b) or Case (c), violating the uniqueness of the generalized Heegaard splitting obtained by weak reduction up to isotopy). Here, $\partial V^{*}$ belongs to the genus-two component of $F-\partial W^{*}$ by Lemma 2.8 and therefore we can take a bandsum of two parallel copies of $V^{*}$ in $\mathcal{V}$ which misses $W^{*}$, say $V^{\prime}$. If we choose the meridian disk of the solid torus which $W^{*}$ cuts off from $\mathcal{W}$ so that it would miss $W^{*}$, 
say $\tilde{W}$, then $\tilde{W}$ also misses $V^{*}$ and $V^{\prime}$. That is, $\left\{V^{\prime}, V^{*}, \tilde{W}, W^{*}\right\}$ forms a 3-simplex in $\mathcal{D}_{\mathcal{V W}}(F)$ and the weak reducing pair $\left(V^{*}, \tilde{W}\right)$ consisting of nonseparating disks must be $(\bar{V}, \bar{W})$ by the previous argument.

If both $V^{*}$ and $W^{*}$ are separating, then we can find a weak reducing pair $(\tilde{V}, \tilde{W})$ consisting of nonseparating disks, where $\tilde{V}$ (resp $\tilde{W}$ ) comes from the meridian disk of the solid torus which $V^{*}$ (resp $W^{*}$ ) cuts off from the corresponding compression body. Here, we can assume that $\widetilde{V} \cup \widetilde{W}$ misses $V^{*} \cup W^{*}$, that is, $\left\{V^{*}, \widetilde{V}, \widetilde{W}, W^{*}\right\}$ forms a 3-simplex in $\mathcal{D}_{\mathcal{V} \mathcal{W}}(F)$. If we apply the previous argument to $(\tilde{V}, \tilde{W})$, then $(\tilde{V}, \tilde{W})$ would be $(\bar{V}, \bar{W})$.

This means that an arbitrary weak reducing pair $\left(V^{*}, W^{*}\right)$ of $(\mathcal{V}, \mathcal{W} ; F)$ belongs to some 3-simplex of the form $\Sigma_{V^{\prime} W^{\prime}}=\left\{V^{\prime}, \bar{V}, \bar{W}, W^{\prime}\right\}$ in $\mathcal{D}_{\mathcal{V W}}(F)$ containing the fixed 1-simplex $\{\bar{V}, \bar{W}\}$, where $V^{\prime} \subset \mathcal{V}$ and $W^{\prime} \subset \mathcal{W}$ are band-sums of two parallel copies of $\bar{V}$ and $\bar{W}$ in $\mathcal{V}$ and $\mathcal{W}$ respectively by Lemma 2.17 .

Claim A $\quad \mathcal{D}_{\mathcal{V W}}(F)=\bigcup_{V^{\prime}, W^{\prime}} \Sigma_{V^{\prime} W^{\prime}}$ for all possible $V^{\prime}$ and $W^{\prime}$.

Proof of Claim A Since $\bigcup_{V^{\prime}, W^{\prime}} \Sigma_{V^{\prime} W^{\prime}} \subset \mathcal{D}_{\mathcal{V W}}(F)$ is obvious, we will prove that every simplex of $\mathcal{D}_{\mathcal{V W}}(F)$ belongs to some $\Sigma_{V^{\prime} W^{\prime}}$.

By definition of $\mathcal{D}_{\mathcal{V W}}(F)$ and the assumption that every weak reducing pair belongs to some $\Sigma_{V^{\prime} W^{\prime}}$, we don't need to consider vertices or 1-simplices in $\mathcal{D}_{\mathcal{V W}}(F)$.

If there is a 2 -simplex $\Delta$ in $\mathcal{D}_{\mathcal{V W}}(F)$, then it must be a $\mathcal{V}$-face or a $\mathcal{W}$-face. Otherwise, we can assume that $\Delta \subset \mathcal{D}_{\mathcal{V}}(F)$ without loss of generality and there must be a vertex in $\mathcal{D}_{\mathcal{W}}(F)$ such that $\Delta$ forms a 3 -simplex in $\mathcal{D}_{\mathcal{V W}}(F)$ together with this vertex by the definition of $\mathcal{D}_{\mathcal{V W}}(F)$. Hence, three vertices of the 3-simplex come from $\mathcal{D}_{\mathcal{V}}(F)$, violating Lemma 2.17. Without loss of generality, suppose that $\Delta$ is a $\mathcal{V}$-face. That is, there is a nonseparating $\mathcal{V}$-disk and a separating $\mathcal{V}$-disk in $\Delta$ by Lemma 2.9. If the $\mathcal{W}$-disk of $\Delta$ is separating, then it cannot cut off (torus) $\times I$ from $\mathcal{W}$ by the uniqueness of the generalized Heegaard splitting obtained by weak reduction (otherwise, the generalized Heegaard splitting obtained by weak reduction along a weak reducing pair containing the $\mathcal{W}$-disk would be that of the symmetric case of Case (b) or Case (c)), that is, it cuts off a solid torus from $\mathcal{W}$. Hence, we can choose a meridian disk $\tilde{W}$ of the solid torus which the $\mathcal{W}$-disk cuts off from $\mathcal{W}$ and it misses three vertices of $\Delta$ by Lemma 2.8. Hence, $\Delta$ and $\tilde{W}$ form a 3 -simplex in $\mathcal{D}_{\mathcal{V W}}(F)$. If the $\mathcal{W}$-disk of $\Delta$ is nonseparating, then the boundary of this $\mathcal{W}$-disk must be contained in the genus-two component which the boundary of the separating $\mathcal{V}$-disk of $\Delta$ cuts off from $F$ by Lemma 2.8 and the boundary of the nonseparating $\mathcal{V}$-disk of $\Delta$ must be contained in the genus-one component by Lemma 2.9. Hence, it is easy to find a band-sum 
of two parallel copies of the $\mathcal{W}$-disk in $\mathcal{W}$ so that it misses the three disks of $\Delta$, say $W^{\prime}$, that is, $\Delta$ and $W^{\prime}$ form a 3 -simplex in $\mathcal{D}_{\mathcal{V W}}(F)$. In any case, $\Delta$ belongs to a 3-simplex $\Sigma$, but $\Sigma$ must contain a weak reducing pair consisting of nonseparating disks by Lemma 2.17. Since the choice of such a weak reducing pair is unique as $(\bar{V}, \bar{W})$ by the previous argument, $\Sigma$ is of the form $\Sigma_{V^{\prime} W^{\prime}}$, leading to the result.

If there is a 3-simplex $\Sigma^{\prime}$ in $\mathcal{D}_{\mathcal{V W}}(F)$, then it must contain a weak reducing pair consisting of nonseparating disks by Lemma 2.17. But we can see that this weak reducing pair must be $(\bar{V}, \bar{W})$ by the previous argument. Therefore, $\Sigma^{\prime}$ is of the form $\Sigma_{V^{\prime} W^{\prime}}$, proving the claim.

We don't need to consider higher-dimensional simplices in $\mathcal{D}_{\mathcal{V W}}(F)$ by Lemma 2.17. This completes the proof of Claim A.

By Claim A, $\mathcal{D}_{\mathcal{V W}}(F)=\bigcup_{V^{\prime}, W^{\prime}} \Sigma_{V^{\prime} W^{\prime}}$ for all possible $V^{\prime}$ and $W^{\prime}$. Hence, we can see that $\mathcal{D}_{\mathcal{V W}}(F) \cap \mathcal{D}_{\mathcal{V}}(F)$ is a star-shaped graph since every 3-simplex $\Sigma_{V^{\prime} W^{\prime}}$ intersects $\mathcal{D}_{\mathcal{V}}(F)$ in an edge and the intersections coming from these 3-simplices have the common vertex $\bar{V}$. The symmetric argument also holds for $\mathcal{D}_{\mathcal{V W}}(F) \cap \mathcal{D}_{\mathcal{W}}(F)$. Moreover, we can see that if $\Sigma_{V^{\prime} W^{\prime}} \neq \Sigma_{V^{\prime \prime} W^{\prime \prime}}$, then $\Sigma_{V^{\prime} W^{\prime}} \cap \Sigma_{V^{\prime \prime} W^{\prime \prime}}$ is either (i) the weak reducing pair $\{\bar{V}, \bar{W}\}$, (ii) the $\mathcal{V}$-face $\left\{V^{\prime}=V^{\prime \prime}, \bar{V}, \bar{W}\right\}$ or (iii) the $\mathcal{W}$-face $\left\{\bar{V}, \bar{W}, W^{\prime}=W^{\prime \prime}\right\}$.

Claim B $\mathcal{D}(F)$ is contractible.

Proof of Claim B Consider $\mathcal{D}(F)$. We have

$$
\mathcal{D}(F)=\mathcal{D}_{\mathcal{V}}(F) \cup \mathcal{D}_{\mathcal{V W}}(F) \cup \mathcal{D}_{\mathcal{W}}(F),
$$

where the following hold:

(1) $\mathcal{D}_{\mathcal{V W}}(F) \cap \mathcal{D}_{\mathcal{V}}(F)$ is a star-shaped graph,

(2) $\mathcal{D}_{\mathcal{V W}}(F) \cap \mathcal{D}_{\mathcal{W}}(F)$ is a star-shaped graph, and

(3) $\mathcal{D}_{\mathcal{V}}(F) \cap \mathcal{D}_{\mathcal{W}}(F)=\varnothing$.

In [16, Section 5], McCullough proved that $\mathcal{D}_{\mathcal{V}}(F)$ and $\mathcal{D}_{\mathcal{W}}(F)$ are contractible (Theorem 2.6) in the sense that they are CW-complexes. Moreover, we can consider $\mathcal{D}_{\mathcal{V W}}(F)$ as a CW-complex. Recall that $\mathcal{D}_{\mathcal{V W}}(F)=\bigcup_{V^{\prime}, W^{\prime}} \Sigma_{V^{\prime} W^{\prime}}$ for all possible $V^{\prime}$ and $W^{\prime}$ by Claim A, where $\Sigma_{V^{\prime} W^{\prime}}$ is the 3 -simplex $\left\{V^{\prime}, \bar{V}, \bar{W}, W^{\prime}\right\}$. Hence, we can construct $\mathcal{D}_{\mathcal{V W}}(F)$ from discrete 0 -cells (the vertices of the two star-shaped graphs $\mathcal{D}_{\mathcal{V W}}(F) \cap \mathcal{D}_{\mathcal{V}}(F)$ and $\mathcal{D}_{\mathcal{V W}}(F) \cap \mathcal{D}_{\mathcal{W}}(F)$ ), followed by 1-cells (consider the edges of each $\Sigma_{V^{\prime} W^{\prime}}$ ), followed by 2-cells (consider the faces of each $\Sigma_{V^{\prime} W^{\prime}}$ ), and finally followed by 3 -cells (consider each $\Sigma_{V^{\prime} W^{\prime}}$ itself) via attaching maps as in the inductive definition of CW-complex. 

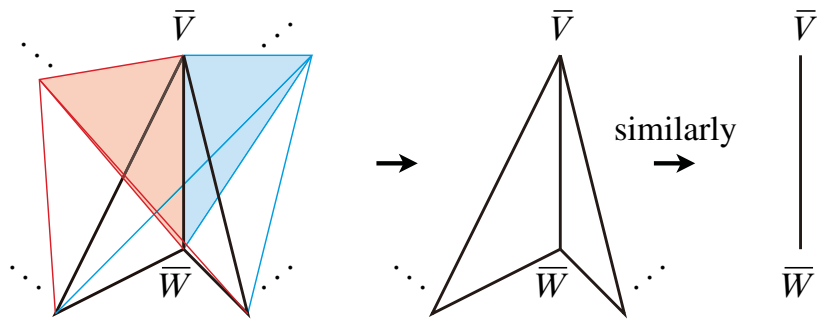

Figure 7: $\mathcal{D}_{\mathcal{V W}}(F)$ is contractible.

First, we prove that $\mathcal{D}_{\mathcal{V W}}(F)$ itself is contractible. It is sufficient to prove that there is a strong deformation-retraction of $\mathcal{D}_{\mathcal{V W}}(F)$ into $\bar{V}$, that is, a continuous map $h: \mathcal{D}_{\mathcal{V W}}(F) \times I \rightarrow \mathcal{D}_{\mathcal{V W}}(F)$ such that (i) $h(x, 0)=x$ for $x \in \mathcal{D}_{\mathcal{V W}}(F)$, (ii) $h(x, 1)=\bar{V}$ for $x \in \mathcal{D}_{\mathcal{V W}}(F)$, and (iii) $h(\bar{V}, t)=\bar{V}$ for $0 \leq t \leq 1$.

Let us consider the star-shaped graph $\mathcal{D}_{\mathcal{V W}}(F) \cap \mathcal{D}_{\mathcal{V}}(F)$. Recall that $\mathcal{D}_{\mathcal{V W}}(F)=$ $\bigcup_{V^{\prime}, W^{\prime}} \Sigma_{V^{\prime} W^{\prime}}$. If we deformation-retract the star-shaped graph $\mathcal{D}_{\mathcal{V W}}(F) \cap \mathcal{D}_{\mathcal{V}}(F)$ into the center point $\bar{V}$ continuously, then each $\Sigma_{V^{\prime} W^{\prime}}$ becomes $\left\{\bar{V}, \bar{W}, W^{\prime}\right\}$ continuously, that is, $\mathcal{D}_{\mathcal{V W}}(F)$ becomes the $\mathcal{W}$-facial cluster containing the weak reducing pair $(\bar{V}, \bar{W})$ continuously (see the first arrow of Figure 7$)$. We take this process as $h: \mathcal{D}_{\mathcal{V W}}(F) \times\left[0, \frac{1}{3}\right] \rightarrow \mathcal{D}_{\mathcal{V W}}(F)$.

Next, if we deformation-retract the star-shaped graph $\mathcal{D}_{\mathcal{V W}}(F) \cap \mathcal{D}_{\mathcal{W}}(F)$ into the center point $\bar{W}$, then the $\mathcal{W}$-facial cluster containing $(\bar{V}, \bar{W})$, similarly, becomes $(\bar{V}, \bar{W})$ continuously (see the second arrow of Figure 7$)$. We take this process as $h: \mathcal{D}_{\mathcal{V W}}(F) \times\left[\frac{1}{3}, \frac{2}{3}\right] \rightarrow \mathcal{D}_{\mathcal{V W}}(F)$.

Finally, if we deformation-retract $(\bar{V}, \bar{W})$ into $\bar{V}$, then it becomes $\bar{V}$ continuously. We take this process as $h: \mathcal{D}_{\mathcal{V W}}(F) \times\left[\frac{2}{3}, 1\right] \rightarrow \mathcal{D}_{\mathcal{V W}}(F)$.

We will use the following lemma.

Lemma 3.6 (Hatcher [10, Exercise 23 of Chapter 0]) A CW-complex is contractible if it is the union of two contractible subcomplexes whose intersection is also contractible.

Then we can see that $\mathcal{D}_{\mathcal{V}}(F) \cup \mathcal{D}_{\mathcal{V W}}(F)$ is also a CW-complex. Moreover, each of $\mathcal{D}_{\mathcal{V}}(F)$ and $\mathcal{D}_{\mathcal{V W}}(F)$ is a subcomplex of $\mathcal{D}_{\mathcal{V}}(F) \cup \mathcal{D}_{\mathcal{V W}}(F)$. Therefore, $\mathcal{D}_{\mathcal{V}}(F) \cup$ $\mathcal{D}_{\mathcal{V W}}(F)$ is contractible by Lemma 3.6 since $\mathcal{D}_{\mathcal{V}}(F)$ is contractible by Theorem 2.6, $\mathcal{D}_{\mathcal{V W}}(F)$ is contractible by the previous observation, and $\mathcal{D}_{\mathcal{V}}(F) \cap \mathcal{D}_{\mathcal{V W}}(F)$ is a star-shaped graph which is contractible. Hence, we can see that $\mathcal{D}(F)=\left(\mathcal{D}_{\mathcal{V}}(F) \cup\right.$ $\left.\mathcal{D}_{\mathcal{V W}}(F)\right) \cup \mathcal{D}_{\mathcal{W}}(F)$ is also contractible by Lemma 3.6.

This completes the proof of Claim B. 
Case (b) $V$ cuts off (torus) $\times I$ from $\mathcal{V}$ and $W$ is nonseparating in $\mathcal{W}$.

In this case, $\partial W$ belongs to the genus-two component of $F-\partial V$ by Lemma 2.8 . Hence, we can take a separating disk $W^{\prime} \subset \mathcal{W}$ by a band-sum of two parallel copies of $W$ such that $W^{\prime}$ misses $V$. Here, we get a 2-simplex $\Delta=\left\{V, W, W^{\prime}\right\}$ in $\mathcal{D}_{\mathcal{V W}}(F)$. Let $\varepsilon$ be the $\mathcal{W}$-facial cluster containing $\Delta$ guaranteed by Lemma 2.15.

The generalized Heegaard splitting obtained by weak reduction along $(V, W)$ consists of two splittings $\left(\mathcal{V}_{1}, \mathcal{V}_{2} ; \bar{F}_{V}\right)$ and $\left(\mathcal{W}_{1}, \mathcal{W}_{2} ; F_{W}\right)$ such that $\partial_{-} \mathcal{V}_{2} \cap \partial_{-} \mathcal{W}_{1}=T$, where $\bar{F}_{V}$ comes from the genus-two component of $F_{V}, \partial_{-} \mathcal{V}_{2}$ consists of a torus, and $\partial_{-} \mathcal{W}_{1}$ consists of two tori.

Let $\mathcal{V}^{\prime}\left(\operatorname{resp} \mathcal{W}^{\prime}\right)$ be the region between $\partial_{+} \mathcal{V}$ and $\bar{F}_{V}$ in $\mathcal{V}$ (resp $\partial_{+} \mathcal{W}$ and $F_{W}$ in $\mathcal{W}$ ). Then we can see that $V$ is a separating compressing disk of $\mathcal{V}^{\prime}$ and $W$ is a nonseparating compressing disk of $\mathcal{W}^{\prime}$. Since $\mathcal{V}^{\prime}$ is a genus-three compression body with negative boundary consisting of a torus and a genus-two surface and $\mathcal{W}^{\prime}$ is a compression body with negative boundary consisting of a genus-two surface, $V$ and $W$ are uniquely determined in $\mathcal{V}^{\prime}$ and $\mathcal{W}^{\prime}$, respectively, up to isotopy. Hence, we get the induced isotopy classes of $V$ and $W$ in $\mathcal{V}$ and $\mathcal{W}$ from the isotopy classes of $V$ and $W$ in $\mathcal{V}^{\prime}$ and $\mathcal{W}^{\prime}$, respectively. Moreover, the uniqueness of the isotopy classes of the embeddings of the thick levels of the generalized Heegaard splitting obtained by weak reduction from $(\mathcal{V}, \mathcal{W} ; F)$ in the relevant compression bodies forces the choice of the induced isotopy classes of $V$ and $W$ in $\mathcal{V}$ and $\mathcal{W}$ to be unique. This means that we can consider the weak reducing pair $(V, W)$ as the fixed one $(\bar{V}, \bar{W})$ even though we've chosen an arbitrary weak reducing pair consisting of a $\mathcal{V}$-disk cutting off (torus) $\times I$ from $\mathcal{V}$ and a nonseparating $\mathcal{W}$-disk.

Let us consider an arbitrary weak reducing pair $\left(V^{*}, W^{*}\right)$ for $(\mathcal{V}, \mathcal{W} ; F)$. If $W^{*}$ cuts off (torus) $\times I$ from $\mathcal{W}$, then the generalized Heegaard splitting obtained by weak reduction along $\left(V^{*}, W^{*}\right)$ would be the symmetric case of Case (b) or Case (c), violating the uniqueness of the generalized Heegaard splitting obtained by weak reduction up to isotopy. Hence, $W^{*}$ does not cut off (torus) $\times I$ from $\mathcal{W}$. Moreover, if $V^{*}$ does not cut off (torus) $\times I$ from $\mathcal{V}$, then $V^{*}$ is nonseparating in $\mathcal{V}$ or it cuts off a solid torus from $\mathcal{V}$, that is, the generalized Heegaard splitting obtained by weak reduction along $\left(V^{*}, W^{*}\right)$ would be that of Case (a) or the symmetric case of Case (b), leading to a contradiction by the same argument. Therefore, $V^{*}$ cuts off (torus) $\times I$ from $\mathcal{V}$. If $W^{*}$ is nonseparating in $\mathcal{W}$, then we take $W^{* *}=W^{*}$. If $W^{*}$ is separating, then we take $W^{* *}$ to be the meridian disk of the solid torus that $W^{*}$ cuts off from $\mathcal{W}$ and $W^{* *} \cap V^{*}=\varnothing$ by Lemma 2.8. If we apply the arguments in the previous paragraph to the weak reducing pair $\left(V^{*}, W^{* *}\right)$, then $\left(V^{*}, W^{* *}\right)$ would be $(\bar{V}, \bar{W})$ and therefore the weak reducing pair $\left(V^{*}, W^{*}\right)$ belongs to the $\mathcal{W}$-facial cluster $\varepsilon$. This means that every weak reducing pair of $(\mathcal{V}, \mathcal{W} ; F)$ belongs to the $\mathcal{W}$-facial cluster $\varepsilon$. 


\section{Claim C $\mathcal{D}_{\mathcal{V W}}(F)=\varepsilon$.}

Proof of Claim C It is sufficient to show that every $\operatorname{simplex}$ of $\mathcal{D}_{\mathcal{V W}}(F)$ belongs to $\varepsilon$.

By definition of $\mathcal{D}_{\mathcal{V W}}(F)$ and the assumption that every weak reducing pair belongs to $\varepsilon$, we don't need to consider vertices or 1-simplices in $\mathcal{D}_{\mathcal{V W}}(F)$.

If we use the same argument in the proof of Claim A, then we can see that if there is a 2 -simplex $\Delta$ in $\mathcal{D}_{\mathcal{V W}}(F)$, then it must be a $\mathcal{V}$-face or a $\mathcal{W}$-face. If $\Delta$ is a $\mathcal{V}$-face, then a $\mathcal{V}$-disk of $\Delta$ cuts off a solid torus from $\mathcal{V}$ and the other $\mathcal{V}$-disk is a meridian disk of the solid torus by Lemma 2.9, that is, the generalized Heegaard splitting obtained by weak reduction along any weak reducing pair in $\Delta$ would be that of Case (a) or the symmetric case of Case (b), violating the uniqueness of the generalized Heegaard splitting obtained by weak reduction up to isotopy.

Hence, $\Delta$ must be a $\mathcal{W}$-face. In this case, the $\mathcal{V}$-disk of $\Delta$, say $V^{\prime}$, must cut off (torus) $\times I$ from $\mathcal{V}$ by the uniqueness of the generalized Heegaard splitting obtained by weak reduction up to isotopy and there is a nonseparating $\mathcal{W}$-disk in $\Delta$ by Lemma 2.9, say $\tilde{W}$. If we use the previous argument, then the weak reducing pair $\left(V^{\prime}, \tilde{W}\right)$ would be $(\bar{V}, \bar{W})$ and therefore $\Delta \subset \varepsilon$, leading to the result.

If there is a 3 -simplex $\Sigma$ in $\mathcal{D}_{\mathcal{V W}}(F)$, then it must contain a $\mathcal{V}$-face by Lemma 2.17, that is, we get a contradiction, similar to the previous $\mathcal{V}$-face case.

We don't need to consider higher-dimensional simplices in $\mathcal{D}_{\mathcal{V W}}(F)$ by Lemma 2.17. This completes the proof of Claim C.

Similarly to Case (a), we can prove that $\mathcal{D}(F)$ is contractible.

Case (c) Each of $V$ and $W$ cuts off (torus) $\times I$ from $\mathcal{V}$ or $\mathcal{W}$, respectively.

The generalized Heegaard splitting obtained by weak reduction along $(V, W)$ consists of two splittings $\left(\mathcal{V}_{1}, \mathcal{V}_{2} ; \bar{F}_{V}\right)$ and $\left(\mathcal{W}_{1}, \mathcal{W}_{2} ; \bar{F}_{W}\right)$ such that $\partial_{-} \mathcal{V}_{2} \cap \partial_{-} \mathcal{W}_{1}=T$, where $\bar{F}_{V}$ and $\bar{F}_{W}$ come from the genus-two components of $F_{V}$ and $F_{W}$, respectively, and both $\partial_{-} \mathcal{V}_{2}$ and $\partial_{-} \mathcal{W}_{1}$ consist of two tori.

Let $\mathcal{V}^{\prime}\left(\right.$ resp $\left.\mathcal{W}^{\prime}\right)$ be the region between $\partial_{+} \mathcal{V}$ and $\bar{F}_{V}$ in $\mathcal{V}$ (resp $\partial_{+} \mathcal{W}$ and $\bar{F}_{W}$ in $\mathcal{W})$. Then we can see that $V$ and $W$ are separating compressing disks of $\mathcal{V}^{\prime}$ and $\mathcal{W}^{\prime}$, respectively. Since $\mathcal{V}^{\prime}$ and $\mathcal{W}^{\prime}$ are genus-three compression bodies with negative boundary consisting of a torus and a genus-two surface, $V$ and $W$ are uniquely determined in $\mathcal{V}^{\prime}$ and $\mathcal{W}^{\prime}$ respectively up to isotopy. Hence, we get the induced isotopy classes of $V$ and $W$ in $\mathcal{V}$ and $\mathcal{W}$ from the isotopy classes of $V$ and $W$ in $\mathcal{V}^{\prime}$ and $\mathcal{W}^{\prime}$, 
respectively. Moreover, the uniqueness of the isotopy classes of the embeddings of the thick levels of the generalized Heegaard splitting obtained by weak reduction from $(\mathcal{V}, \mathcal{W} ; F)$ in the relevant compression bodies forces the choice of the induced isotopy classes of $V$ and $W$ in $\mathcal{V}$ and $\mathcal{W}$ to be unique. This means that we can consider the weak reducing pair $(V, W)$ as the fixed one $(\bar{V}, \bar{W})$ even though we've chosen an arbitrary weak reducing pair consisting of disks which cut off (torus) $\times I$ 's in the relevant compression bodies.

Let us consider an arbitrary weak reducing pair $\left(V^{*}, W^{*}\right)$ for $(\mathcal{V}, \mathcal{W} ; F)$. If one of $V^{*}$ and $W^{*}$ does not cut off (torus) $\times I$ from $\mathcal{V}$ or $\mathcal{W}$, then the generalized Heegaard splitting obtained by weak reduction along $\left(V^{*}, W^{*}\right)$ would be that of Case (a) or (possibly the symmetric case of) Case (b), violating the uniqueness of the generalized Heegaard splitting obtained by weak reduction up to isotopy. Therefore, $V^{*}$ and $W^{*}$ must cut off (torus) $\times I$ from $\mathcal{V}$ and $\mathcal{W}$, respectively. If we apply the arguments in the previous paragraph to the weak reducing pair $\left(V^{*}, W^{*}\right)$, then $\left(V^{*}, W^{*}\right)$ would be $(\bar{V}, \bar{W})$. Hence, $\mathcal{D}_{\mathcal{V W}}(F)$ is just $(\bar{V}, \bar{W})$ itself.

Therefore, $\mathcal{D}(F)$ is contractible. This completes the proof of Lemma 3.5.

Now we deal with the case where the inner thin level consists of two tori.

Lemma 3.7 Let $(\mathcal{V}, \mathcal{W} ; F)$ be a weakly reducible unstabilized genus-three Heegaard splitting in an orientable, irreducible 3-manifold. If every weak reducing pair of $F$ gives the same generalized Heegaard splitting obtained by weak reduction up to isotopy such that the inner thin level consists of two tori and the embedding of each thick level in the relevant compression body is unique up to isotopy, then $\mathcal{D}(F)$ is contractible.

Proof Let us consider a weak reducing pair $(V, W)$ for $(\mathcal{V}, \mathcal{W} ; F)$. Here, $V$ and $W$ are nonseparating but $\partial V \cup \partial W$ is separating in $F$ as we checked in the proof of Lemma 2.16 by the assumption that the inner thin level consists of two tori. This means that $\partial V \cup \partial W$ cuts off two twice-punctured tori from $F$. The generalized Heegaard splitting obtained by weak reduction along $(V, W)$ consists of two splittings $\left(\mathcal{V}_{1}, \mathcal{V}_{2} ; F_{V}\right)$ and $\left(\mathcal{W}_{1}, \mathcal{W}_{2} ; F_{W}\right)$ such that $\partial_{-} \mathcal{V}_{2}=\partial_{-} \mathcal{W}_{1}=F_{V W}=T_{1} \cup T_{2}$, where each $T_{i}$ is a torus.

Let $\mathcal{V}^{\prime}\left(\operatorname{resp} \mathcal{W}^{\prime}\right)$ be the region between $\partial_{+} \mathcal{V}$ and $F_{V}$ in $\mathcal{V}\left(\operatorname{resp} \partial_{+} \mathcal{W}\right.$ and $F_{W}$ in $\left.\mathcal{W}\right)$. Then we can see that $V$ and $W$ are nonseparating compressing disks of $\mathcal{V}^{\prime}$ and $\mathcal{W}^{\prime}$, respectively. Since $\mathcal{V}^{\prime}$ and $\mathcal{W}^{\prime}$ are genus-three compression bodies with negative boundary consisting of a genus-two surface, $V$ and $W$ are uniquely determined in $\mathcal{V}^{\prime}$ and $\mathcal{W}^{\prime}$, respectively, up to isotopy. Hence, we get the induced isotopy classes of $V$ and $W$ in $\mathcal{V}$ and $\mathcal{W}$ from the isotopy classes of $V$ and $W$ in $\mathcal{V}^{\prime}$ and $\mathcal{W}^{\prime}$, respectively. 
Moreover, the uniqueness of the isotopy classes of the embeddings of the thick levels of the generalized Heegaard splitting obtained by weak reduction from $(\mathcal{V}, \mathcal{W} ; F)$ in the relevant compression bodies forces the choice of the induced isotopy classes of $V$ and $W$ in $\mathcal{V}$ and $\mathcal{W}$ to be unique. This means that we can consider the weak reducing pair $(V, W)$ as the fixed one $(\bar{V}, \bar{W})$ even though we've chosen an arbitrary weak reducing pair. Hence, $\mathcal{D}_{\mathcal{V W}}(F)$ is just $(\bar{V}, \bar{W})$ itself.

Therefore, $\mathcal{D}(F)$ is contractible; the argument is similar to Case (c) of Lemma 3.5. This completes the proof.

Lemma 3.1, Lemma 3.4, Lemma 3.5 and Lemma 3.7 prove Theorem 1.1.
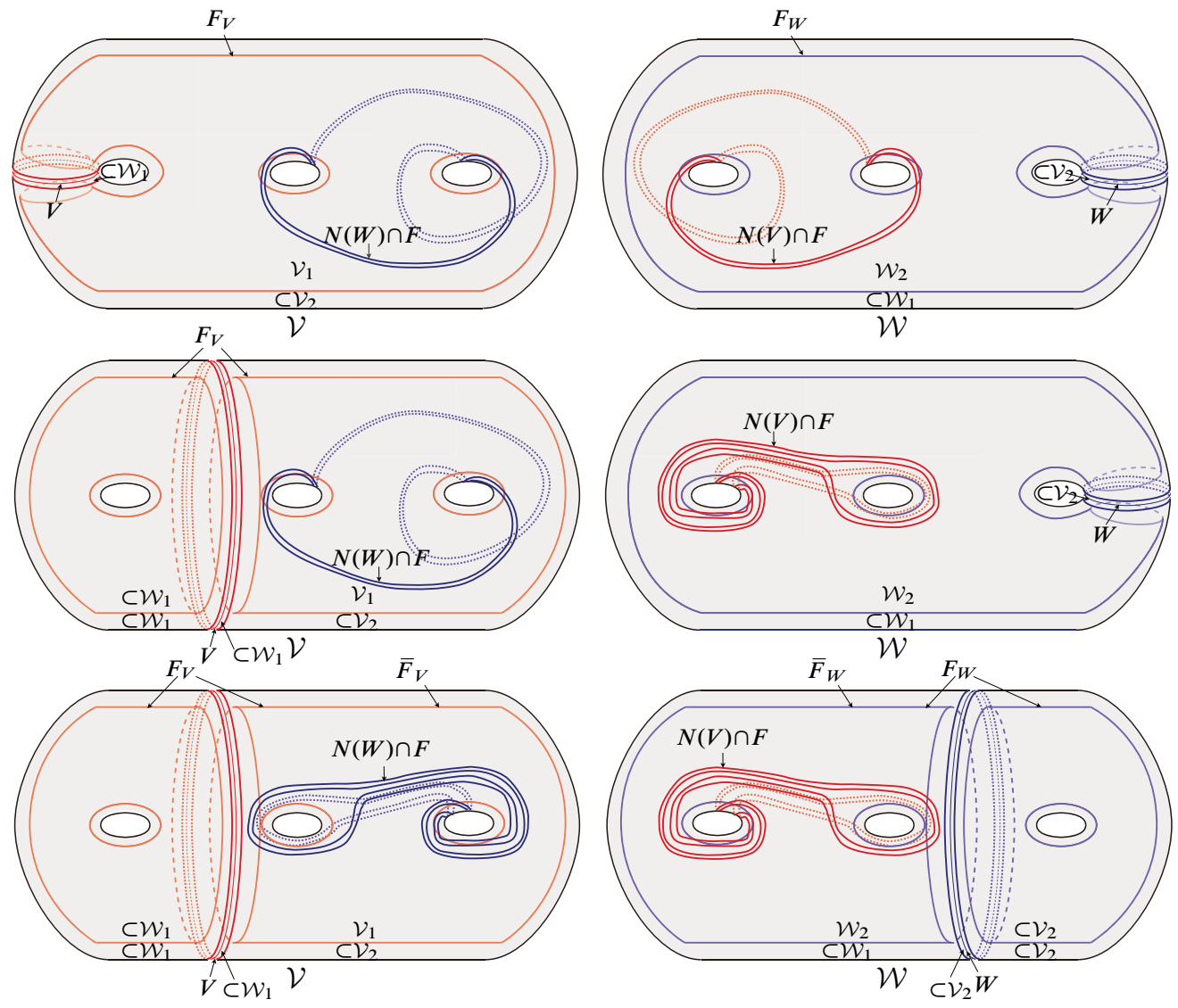

Figure 8: The GHSs for Figure 6(a). (We omit the symmetric case for the second figure.) 


\section{Appendix: The GHSs obtained by preweak reductions}

We give descriptive figures of all possible cases of the GHSs obtained by preweak reductions from an unstabilized genus three Heegaard splitting $(\mathcal{V}, \mathcal{W} ; F)$ of an irreducible 3-manifold $M$, where these preweak reductions give GHSs of the form $\left(\mathcal{V}_{1}, \mathcal{V}_{2}\right) \cup\left(\mathcal{W}_{1}, \mathcal{W}_{2}\right)$ for $\partial_{-} \mathcal{V}_{2} \cap \partial_{-} \mathcal{W}_{1} \neq \varnothing$. Note that we only consider the case $\partial_{-} \mathcal{V}_{1}=\partial_{-} \mathcal{W}_{2}=\varnothing$ because $\partial_{-} \mathcal{V}_{1}$ and $\partial_{-} \mathcal{W}_{2}$ do not affect the shape of $\mathcal{V}_{2}$ and $\mathcal{W}_{1}$ and the inner thin level.

Note that $N(V)$ and $N(W)$ in Figures 8-11 mean product neighborhoods of $V$ and $W$ in $\mathcal{V}$ and $\mathcal{W}$, respectively.
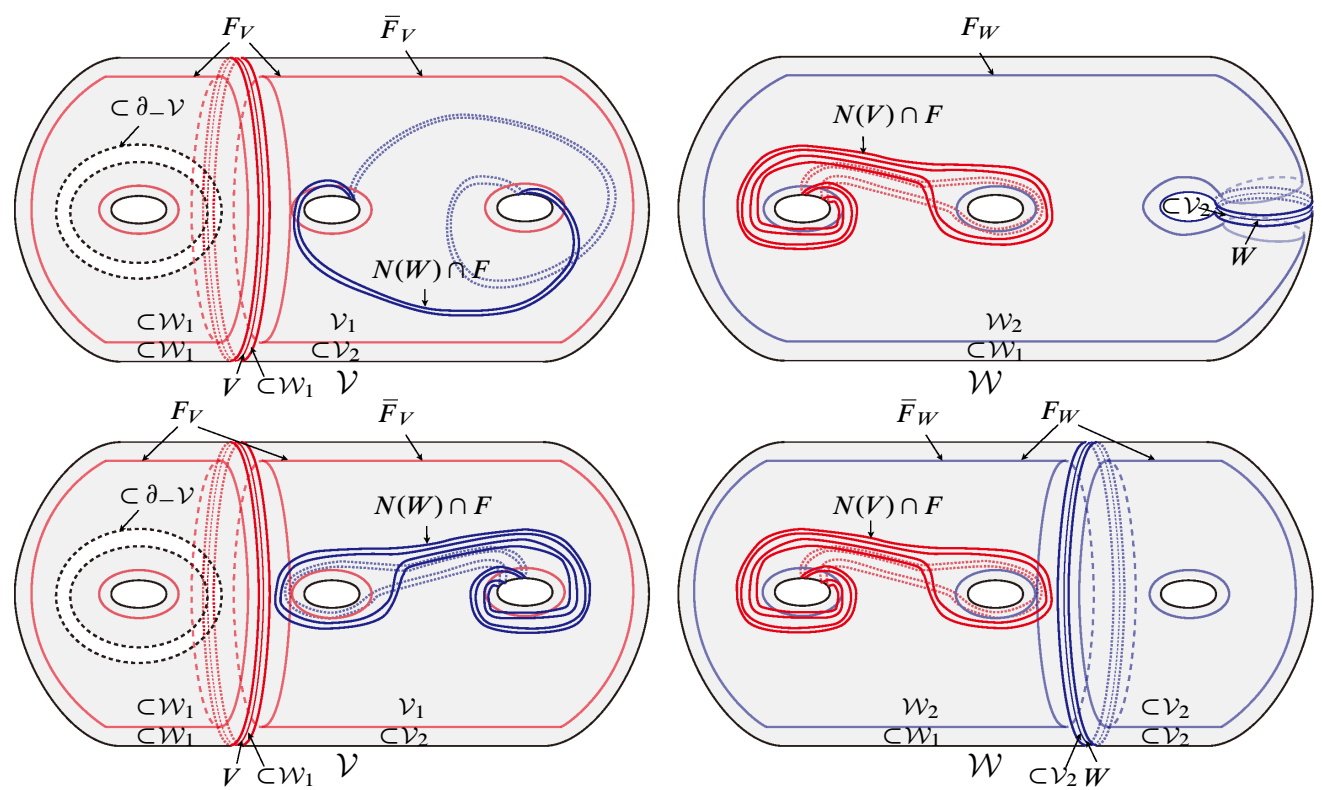

Figure 9: The GHSs for Figure 6(b). (We omit the symmetric cases where $W$ cuts off (torus) $\times I$ from $\mathcal{W}$.)
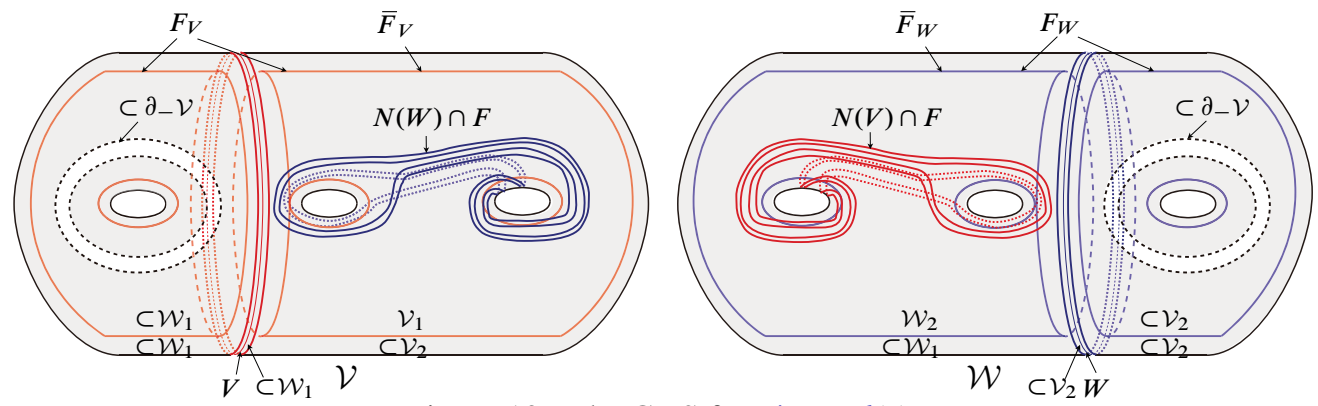

Figure 10: The GHS for Figure 6(c). 

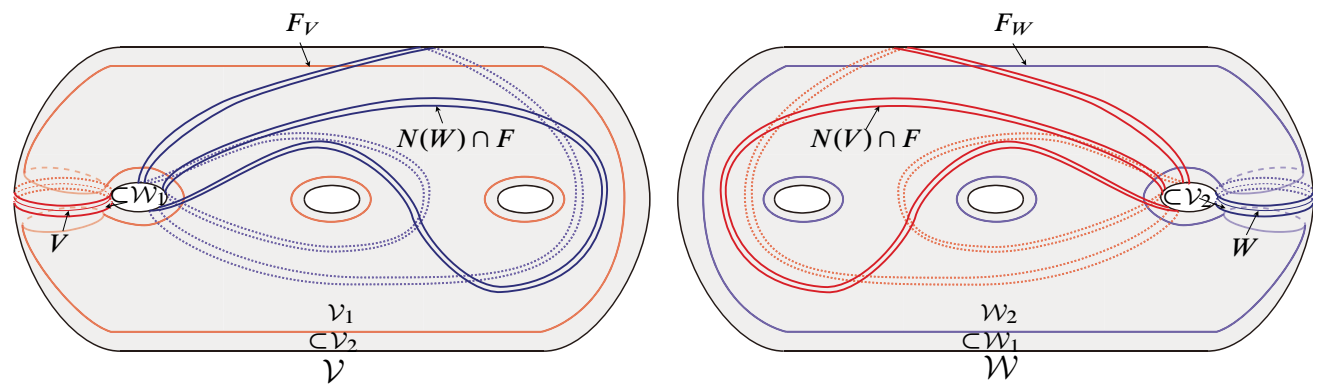

Figure 11: The GHS for Figure 4.

\section{References}

[1] D Bachman, Critical Heegaard surfaces, Trans. Amer. Math. Soc. 354 (2002) 40154042 MR1926863

[2] D Bachman, Connected sums of unstabilized Heegaard splittings are unstabilized, Geom. Topol. 12 (2008) 2327-2378 MR2443968

[3] D Bachman, Topological index theory for surfaces in 3-manifolds, Geom. Topol. 14 (2010) 585-609 MR2602846

[4] D Bachman, Normalizing topologically minimal surfaces, I: Global to local index (2012) arXiv:1210.4573

[5] D Bachman, Normalizing topologically minimal surfaces, II: Disks (2012) arXiv: 1210.4574

[6] D Bachman, Normalizing topologically minimal surfaces, III: Bounded combinatorics (2013) arXiv:1303.6643

[7] D Bachman, Stabilizing and destabilizing Heegaard splittings of sufficiently complicated 3-manifolds, Math. Ann. 355 (2013) 697-728 MR3010144

[8] D Bachman, J Johnson, On the existence of high index topologically minimal surfaces, Math. Res. Lett. 17 (2010) 389-394 MR2653676

[9] Q E, F Lei, Critical Heegaard surfaces obtained by self-amalgamation, J. Knot Theory Ramifications 22 (2013) 1350015, 7 MR3069753

[10] A Hatcher, Algebraic topology, Cambridge Univ. Press, Cambridge (2002) MR1867354

[11] A Ido, Y Jang, T Kobayashi, Heegaard splittings of distance exactly $n$, Algebr. Geom. Topol. 14 (2014) 1395-1411 MR3190598

[12] J Kim, On critical Heegaard splittings of tunnel number two composite knot exteriors, J. Knot Theory Ramifications 22 (2013) 1350065, 11 MR3143581

[13] J Kim, On unstabilized genus three critical Heegaard surfaces, Topology Appl. 165 (2014) 98-109 MR3174071 
[14] J H Lee, Critical Heegaard surfaces obtained by amalgamation, Topology Appl. 160 (2013) 111-116 MR2995082

[15] M Lustig, Y Moriah, Closed incompressible surfaces in complements of wide knots and links, Topology Appl. 92 (1999) 1-13 MR1670164

[16] D McCullough, Virtually geometrically finite mapping class groups of 3-manifolds, J. Differential Geom. 33 (1991) 1-65 MR1085134

[17] T Saito, M Scharlemann, J Schultens, Lecture notes on generalized Heegaard splittings (2005) arXiv:math/0504167

[18] M Scharlemann, A Thompson, Thin position for 3-manifolds, from: "Geometric topology", (C Gordon, Y Moriah, B Wajnryb, editors), Contemp. Math. 164, Amer. Math. Soc. (1994) 231-238 MR1282766

[19] J Schultens, Additivity of tunnel number for small knots, Comment. Math. Helv. 75 (2000) 353-367 MR1793793

BK21 PLUS SNU Mathematical Sciences Division, Seoul National University 1 Gwanak-ro, Gwanak-Gu, Seoul 08826, South Korea

pibonazi@gmail.com

Received: 18 August 2014 Revised: 25 July 2015 
